\title{
Control analysis of trophic chains
}

\author{
Wayne M. Getz ${ }^{\mathrm{a}, \mathrm{b}, *}$, Hans V. Westerhoff ${ }^{\mathrm{a}, \mathrm{c}}$, \\ Jan-Hendrik S. Hofmeyr ${ }^{\text {d }}$, Jacky L. Snoep ${ }^{\text {c,d }}$ \\ a Stellenbosch Institute for Advanced Study, Private Bag X1, Matieland 7602, South Africa \\ ${ }^{\mathrm{b}}$ Department of Environmental Science, Policy, and Management, University of California at Berkeley, Berkeley, CA 94720-3112, USA \\ ${ }^{c}$ BioCentrum Amsterdam, De Boelelaan 1087, NL-1081 HV Amsterdam, The Netherlands \\ ${ }^{\mathrm{d}}$ Department of Biochemistry, University of Stellenbosch, Private Bag X1, Matieland 7602, South Africa
}

Received 30 July 2002; received in revised form 7 May 2003; accepted 10 June 2003

\begin{abstract}
Inspired by the application of metabolic control analysis (MCA) to biochemical networks, we conduct a generalized sensitivity analysis of the equilibrium of a set of differential equations used to model trophic chains. We focus on changes in the equilibrium to perturbations of feeding (i.e. functional responses) and growth rate functions. So-called control coefficient connectivity relationships are derived for two broad classes of trophic chains: those governed by linear and those governed by nonlinear growth functions. These connectivity relationships link global sensitivity coefficients to local elasticities represented by the normalized partial derivatives of the rate functions. We derive results for specific classes of trophic chains, including hyperbolic growth functions used in metaphysiological models. Our results provide formulae for computing the degree to which control by the feeding and growth functions is top-down versus bottom-up at any level in any given trophic chain. Control analysis provides a framework for articulating the degree to which equilibrium—or, more usefully, long term average—population levels are influenced by the different rate functions in terms of local elasticity functions. Control Analysis also provides techniques for probing the trophic cascade hypothesis.
\end{abstract}

(C) 2003 Elsevier B.V. All rights reserved.

Keywords: Bottom-up top-down control; Foodwebs; Metabolic control analysis; Sensitivity analysis; Functional response

\section{Introduction}

Biological systems are often represented as networks of nonlinearly interacting objects or nodes. The interaction of these objects is modeled using rate equations that allow the behavior of the system to be integrated or simulated and studied as a whole. This strategy has been applied at vastly different levels of organization, ranging from biochemical networks within cells to networks of interacting populations constituting ecosystems. A question that universally

\footnotetext{
* Corresponding author. Fax: +1-510-642-7428.

E-mail address: getz@nature.berkeley.edu (W.M. Getz).
}

applies to such networks is: "What are the consequences for the objects of the network if one or more of the functions (processes) connecting any two of them is perturbed."

A class of methods used to answer such questions falls under the rubric of sensitivity analysis. Sensitivity of system trajectories or other characterizing values to changes in the value of specific system parameters has been of considerable interest to ecologists (e.g. see Swartzman and Kaluzny, 1987; Caswell, 2000). Biochemists, on the other hand, have focused on the sensitivity of particular system process rates to perturbations in process rates in other parts of the biochemical system. They have developed coherent theories for 
undertaking this type of sensitivity analysis, that have been classified into two related methods of analysis respectively know as metabolic control analysis (MCA) (Kacer and Burns, 1973; Heinrich and Rapoport, 1974; Giersch, 1988a,b; Kahn and Westerhoff, 1991; Fell, 1996; Hofmeyr and Cornish-Bowden, 1996; Hofmeyr and Westerhoff, 2001; Hofmeyr, 2001) and biochemical systems theory (BST) (Savageau, 1976, 1996).

MCA methods of analysis have served to identify principles of control and regulation that are generalizable to other networks, such as foodwebs and energy flows in ecosystems (Wennekers and Giersch, 1991; Giersch, 1991, 1995), but these generalizations were not couched in terms of the per-capita process rates driving foodwebs. Unlike biochemical processes which are generally cast in terms of total process rates, ecological systems are dominated by per-capita process rates-specifically, per-capita rates at which individuals extract resources as a function of resource and species densities, and per-capita growth rates as a function of resource consumption rates (e.g. see Getz, 1991, 1993, 1994). This difference in how we view biochemical and ecological processes reflects the fact that the density of chemical species is controlled by interspecific synthetic processes rather than intraspecific reproductive processes.

In this paper, we focus on developing a theory of process control in the topologically simplest of all foodwebs - the unbranched trophic chain-using MCA as guide to our basic method of analysis. Trophic chains are based on an autotroph utilizing an energy flux to sustain itself over many generations while exploiting a renewable resource (e.g. plants or algae using sunlight to grow in the presence of soil nutrients and water). The next link in the chain is a herbivore (or phytoplanktivore in aquatic planktonic systems), followed by primary and possibly secondary and tertiary carnivores, depending on the length of the food chain.

The fact that long term changes in the density of a population at one level in a trophic chain may have repercussions for populations at several other levels in that chain was first articulated by Hairston et al. (1960). They formulated what has now become know as the Green World Hypothesis in which they maintained that the world can only be green in places where carnivores keep the herbivores in check from over-exploiting the plants below them. This hypothesis was generalized by Oksanen et al. (1981) and Fretwell $(1977,1987)$ to systems with five trophic levels. The general hypothesis that foodwebs exhibit responses at the primary producer level when perturbations occur to populations two levels up (i.e. the primary carnivore level in plant-herbivore-carnivore systems) was coined the trophic cascade hypothesis by Paine (1980). This hypothesis, as articulated by Brett and Goldman (1996), holds that changes in density at each trophic level of a foodweb are inversely related to changes in density at the trophic level above and directly related to changes in density at the trophic level below. The widespread validity of this hypothesis has been documented in a meta-analysis conducted by Schmitz et al. (2000) on data from 41 different studies.

Discussions why, if at all, the trophic cascade hypothesis should be true have been confounded by insufficient attention to differences between the concepts of 'species level cascades' and 'community level cascades' (Polis, 1999). Species level cascades pertain to trophic chains embedded within foodwebs. Such trophic chains, however, are rarely sufficiently isolated from other parts of the foodweb to allow the response of the species in a particular chain to be considered in isolation from the rest of the foodweb, although species level cascades have been identified in systems where omnivory blurs the trophic structure (Power, 1990). Community level cascades, on the other hand, require that foodwebs have well-identified trophic levels (which, for example, become fuzzy when omnivory is prevalent-e.g. see Persson et al., 1996) and that responses are measured as an aggregate of the response of all populations functioning at the same trophic level. Further, even with regard to trophic chains that appear to be relatively isolatable from a more extensive web, discussions on the relative importance of top-down (predator) control versus bottom-up (resource) control lead to a polarization that control resides in one or the other, rather than the notion that control is distributed over both.

In this paper we resolve the debate on top-down versus bottom-up control by using a trophic chain model to demonstrate how control is distributed over the different levels for both species and community level cascades (in the former case, the model variables represent species densities; in the latter case, they represent the aggregate of all species densities at 
each of the trophic levels) rather than residing at any privileged level. We also develop a method of analysis and a vocabulary for talking about how we might expect steady states in trophic cascades to change in response to perturbations in trophic and population processes. In reality, biological populations fluctuate around some average level that generally has a cyclic or seasonal component. In such cases, the analysis presented here must be interpreted in the context of trophic chains that can equilibrate within a season or in terms of population levels averaged over appropriately long intervals of time.

Beginning with the pioneering work of Hairston et al. (1960), ecologists have examined the extent to which primary producer biomass is influenced by resources needed for growth versus the extraction of biomass by consumers (Oksanen et al., 1981; Fretwell, 1987; Power, 1992; Polis and Strong, 1996). Ecologists have set up experiments and conducted field work to address this question, but the more subtle issues relating to this question have never been formally articulated using mathematical analysis.

The material presented below closes this gap. We call the approach trophic control analysis (TCA). Although TCA has much in common with MCA, trophic chains are fundamentally different from metabolic networks. For TCA to obtain results relevant to trophic chains it needs to move beyond MCA (some details can be found in our companion paper). For example, trophic chains cannot simply be couched in terms of populations connected by mass flow processes, as defined by a stoichiometry matrix (Giersch, 1995). Unlike biochemical networks, growth processes are generally nonconservative (biomass is lost to excretion and deaths as material passes up the food chain) in a way that corresponds to slippage in complex biochemical networks (Westerhoff and Van Dam, 1987; Schuster and Westerhoff, 1999).

\section{Model and methods}

\subsection{Trophic model}

The history of trophic chain models is rooted in the prey-predator models of Lotka (1925) and Volterra (1926) with extensions due, among others, to the ideas of Leslie (1948), Holling (1959), Rosenzweig and MacArthur (1963), and Oksanen et al. (1981). A more recent view of trophic chains that focuses on the process of per-capita feeding (alternatively foraging, extraction, ingestion) and growth process rates results in the following general model (Getz, 1991, 1993, 1994). Define $x_{i}, i=1, \ldots, n$ as the biomass in the $i$ th level of an $n$-level trophic chain. Assume the lowest level of the chain $(i=1)$ is supported by an underlying buffered resource $x_{0}$ and the highest level $(i=n)$ is subject to extraction by a process modeled by an external input or driving function (e.g. human harvest). Further, define $g_{i}$ to be the growth rate of each trophic level and $f_{i+1}$ the per-capita rate at which biomass at the $i$ th trophic level is extracted by each unit of biomass at the $(i+1)$ th trophic level. The model for the dynamics of the $i$ th trophic level takes the form

$$
\frac{\mathrm{d} x_{i}}{\mathrm{~d} t}=g_{i}\left(f_{i}\right) x_{i}-f_{i+1} x_{i+1}, \quad i=1, \ldots, n .
$$

The functions $f_{i}$ are commonly called the functional responses, but we prefer to call them the feeding (or extraction) rate functions. The model, as written, implies that the per-capita growth rate depends on the per-capita feeding rate. Unless otherwise stated, we assume that feeding rates are functions only of the "consumer's" own density $x_{i}$ that of its "resource" $x_{i-1}$-that is, $f_{i}\left(x_{i-1}, x_{i}\right)$. Note, the system can be truncated at $n$ variables $x_{i}, i=1, \ldots, n$, by defining $h=f_{n+1} x_{i+1}$ to represent extraction by a "harvest" rate $h$ that can be set to 0 . Finally, we note that Eq. (1) provides a richer, more naturally framework for incorporating additional resolution in the model pertaining to physiological and behavioral processes than modified Lotka-Volterra approaches such as the Rosenzweig-MacArthur formulation (also see Ramos-Jiliberto et al., 2002).

\subsection{Elasticity and control}

MCA theorems and analysis are usually cast in the context of models that have the form (Heinrich et al., 1977; Reder, 1988; Hofmeyr and Westerhoff, 2001; also see Fell, 1996; Hofmeyr, 2001)

$$
\frac{\mathrm{d} x_{i}}{\mathrm{~d} t}=\sum_{j=1}^{m} n_{i j} v_{j}(\boldsymbol{x}, \lambda), \quad i=1, \ldots, n,
$$


where $\boldsymbol{x}=\left(x_{1}, \ldots, x_{n}\right)^{\prime}\left({ }^{\prime}\right.$ denotes vector transpose $)$ is a metabolite concentration vector, $n_{i j}$ is the $i$ th row and $j$ th column entry of a $n \times m$ stoichiometry matrix $N, \boldsymbol{v}(\boldsymbol{x}, \boldsymbol{\lambda})=\left(v_{1}\left(\boldsymbol{x}, \lambda_{1}\right), \ldots, v_{m}\left(\boldsymbol{x}, \lambda_{m}\right)\right)^{\prime}$ is a vector of process total (as opposed to per capita) rate functions, and $\lambda=\left(\lambda_{1}, \ldots, \lambda_{m}\right)^{\prime}$ is vector of parameters. The functions $v_{j}\left(\boldsymbol{x}, \lambda_{j}\right)$ are assumed to be homogeneous of degree 1 in $\lambda_{j}$ so that the unperturbed state corresponds to $\lambda_{j}=1$. This implies that $\lambda_{j}$ is functionally equivalent to a scaling perturbation of $v_{j}$ and can always be introduced when needed.

MCA focuses on how an unperturbed nontrivial steady-state solution $\hat{\boldsymbol{x}}$ to Eq. (2), that is a solution satisfying

$N \hat{\boldsymbol{v}}=0$,

where $\hat{\boldsymbol{v}}=\boldsymbol{v}(\hat{\boldsymbol{x}}, \mathbf{1})$, responds to perturbations of $\lambda$ around the nominal value $\lambda=1$. Although, from a mathematical point of view, $\hat{\boldsymbol{x}}$ is referred to as an equilibrium, the terminology "steady-state" is used to emphasize the fact that, in general, each of the elements $v_{j}\left(\hat{\boldsymbol{x}}, \lambda_{j}\right)$ are themselves non-zero, but represent steady-state fluxes.

In MCA, each process that occurs at a non-zero rate $v_{j}$ is characterized by elasticity coefficients $\varepsilon_{x_{i}}^{v_{j}}, i=$ $1, \ldots, n, j=1, \ldots, m$ that are defined in terms of the normalized partial derivatives of the process functions $v_{j}$ with respect to the arguments $x_{i}$ : viz.,

$\varepsilon_{x_{i}}^{v_{j}}=\left.\left.\frac{x_{i}}{v_{j}} \frac{\partial v_{j}}{\partial x_{i}}\right|_{\boldsymbol{x}=\hat{\boldsymbol{x}}, \lambda=\mathbf{1}} \equiv \frac{\partial \ln \left|v_{j}\right|}{\partial \ln x_{i}}\right|_{\boldsymbol{x}=\hat{\boldsymbol{x}}, \lambda=\mathbf{1}}$.

Also, in MCA, the control that all the processes exercise on the steady-state concentrations of the entire system is characterized by concentration-control coefficients defined, assuming $x_{i}>0$, by

$c_{v_{j}}^{x_{i}}=\left.\frac{\lambda_{j}}{x_{i}} \frac{\mathrm{d} x_{i}}{\mathrm{~d} \lambda_{j}} \equiv \frac{\mathrm{d} \ln x_{i}}{\mathrm{~d} \ln \lambda_{j}}\right|_{x=\hat{x}, \lambda=1}$.

To facilitate the development of TCA, we replace the feeding and growth functions in Eq. (1) with $f_{j} \rightarrow \lambda_{j} f_{j}$ and $g_{j} \rightarrow \mu_{j} g_{j}$, allowing us to nominally perturb process rates by varying $\lambda_{j}$ or $\mu_{j}$ around the values $\lambda_{j}=1$ or $\mu_{j}=1$. In particular, we use perturbations of this $\lambda_{j}$ to calculate the $n$ TCA feeding control coefficients

$c_{f_{j}}^{x_{i}}=\left.\frac{\mathrm{d} \ln x_{i}}{\mathrm{~d} \ln \lambda_{j}}\right|_{x=\hat{x}(1), \lambda_{j}=1}, \quad i=1, \ldots, n$.
Similarly, the same kind of perturbations can be used to calculate the $n$ TCA growth control coefficients

$c_{g_{j}}^{x_{i}}=\left.\frac{\mathrm{d} \ln x_{i}}{\mathrm{~d} \ln \mu_{j}}\right|_{x=\hat{x}(1), \mu_{j}=1}, \quad i=1, \ldots, n$.

Note that each perturbation of a particular feeding or growth function is made, assuming that the other functions remain unperturbed except through the variation of their arguments. From this perspective, the new steady-state level $\hat{\boldsymbol{x}}$ is considered a function of the particular perturbed growth or feeding function so that for some particular value of $j=1, \ldots, n$, this new steady-state value can be approximated by the expansion

$\hat{\boldsymbol{x}}\left(\lambda_{j}\right) \approx \hat{\boldsymbol{x}}(1)+\left(\lambda_{j}-1\right) c_{f_{j}}^{x_{i}} \frac{\hat{\boldsymbol{x}}(1)}{\lambda_{j}}$

or by

$\hat{\boldsymbol{x}}\left(\mu_{j}\right) \approx \hat{\boldsymbol{x}}(1)+\left(\mu_{j}-1\right) c_{g_{j}}^{x_{i}} \frac{\hat{\boldsymbol{x}}(1)}{\mu_{j}}$,

as the case may be.

\subsection{Feeding and growth functions}

The feeding functions $f_{i}$ in Eq. (1) are typically monotonically increasing, but saturating functions of the associated resource $x_{i-1}$ (see Holling, 1959 and also Table 1), although some ecologists argue that feeding functions might more appropriately depend on the ratio of consumers to resources (i.e. $f_{i}=$ $f_{i}\left(x_{i} / x_{i-1}\right)$-see Arditi and Ginzburg, 1989; Matson and Berryman, 1992; but see Oksanen et al., 1992; Abrams, 1994). More generally, we develop our theory for feeding functions that depend on both the densities of consumers and their resources (i.e. $f_{i}=$ $\left.f_{i}\left(x_{i-1}, x_{i}\right)\right)$. Of course, feeding functions may depend on other species in the hierarchy, but this level of generality is beyond the scope of our analysis.

In addition to the functions listed in Table 1, we present their elasticity coefficients defined by Eq. (4). These coefficients provide one way of characterizing the form of the dependence of the functions on each of the parameters. The elasticity coefficient for a power function is equal to the power to which the variable is raised in the function (see second row in Table 1). 
Table 1

Feeding functions

\begin{tabular}{|c|c|c|}
\hline Name & Form $(i=1,2)$ & Elasticities \\
\hline Lotka-Volterra & $f_{i}\left(x_{i-1}, x_{i}\right)=a_{i} x_{i-1}, \quad a_{i}>0$ & $\varepsilon_{x_{i-1}}^{f_{i}}=1, \quad \varepsilon_{x_{i}}^{f_{i}}=0$ \\
\hline Compensatory power function & $f_{i}\left(x_{i-1}, x_{i}\right)=a_{i} x_{i-1}^{p_{i}}, \quad a_{i}>0,0<p_{i}<1$ & $\varepsilon_{x_{i-1}}^{f_{i}}=p_{i}, \quad \varepsilon_{x_{i}}^{f_{i}}=0$ \\
\hline Holling type II (Holling, 1959) & $f_{i}\left(x_{i-1}, x_{i}\right)=\frac{a_{i} x_{i-1}}{b_{i}+x_{i-1}}, \quad a_{i}>0, b_{i}>0$ & $\varepsilon_{x_{i-1}}^{f_{i}}=\frac{b_{i}}{b_{i}+x_{i-1}}, \quad \varepsilon_{x_{i}}^{f_{i}}=0$ \\
\hline $\begin{array}{l}\text { Ratio-dependent type II } \\
\quad(\text { Getz, 1984) }\end{array}$ & $f_{i}\left(x_{i-1}, x_{i}\right)=\frac{a_{i} x_{i-1}}{b_{i} x_{i}+x_{i-1}}, \quad a_{i}>0, b_{i}>0$ & $\varepsilon_{x_{i-1}}^{f_{i}}=\frac{b_{i} x_{i}}{b_{i} x_{i}+x_{i-1}}, \quad \varepsilon_{x_{i}}^{f_{i}}=\frac{-b_{i} x_{i}}{b_{i} x_{i}+x_{i-1}}$ \\
\hline $\begin{array}{l}\text { Beddington type II } \\
\text { (Beddington, 1975; } \\
\text { DeAngelis et al., 1975) }\end{array}$ & $f_{i}\left(x_{i-1}, x_{i}\right)=\frac{a_{i} x_{i-1}}{b_{i}+x_{i-1}+c_{i} x_{i}}, \quad a_{i}>0, b_{i}>0, c_{i}>0$ & $\begin{aligned} \varepsilon_{x_{i-1}}^{f_{i}} & =\frac{b_{i}+c_{i} x_{i}}{b_{i}+c_{i} x_{i}+x_{i-1}}, \\
\varepsilon_{x_{i}}^{f_{i}} & =\frac{-c_{i} x_{i}}{b_{i}+c_{i} x_{i}+x_{i-1}}\end{aligned}$ \\
\hline $\begin{array}{l}\text { Generalized holling type } \\
\text { III (Getz, 1999) }\end{array}$ & $f_{i}\left(x_{i-1}, x_{i}\right)=\frac{a_{i} x_{i-1}^{\gamma_{i}}}{b_{i}^{\gamma_{i}}+x_{i-1}^{\gamma_{i}}}, \quad a_{i}>0, b_{i}>0, \gamma_{i}>1$ & $\varepsilon_{x_{i-1}}^{f_{i}}=\frac{\gamma_{i} b_{i}^{\gamma_{i}}}{b_{i}^{\gamma_{i}}+x_{i-1}^{\gamma_{i}}}, \quad \varepsilon_{x_{i}}^{f_{i}}=0$ \\
\hline $\begin{array}{l}\text { Generalized holling I-II } \\
\text { hybrid (Getz, 1999) }\end{array}$ & $\begin{array}{l}f_{i}\left(x_{i-1}, x_{i}\right)=\frac{a_{i} x_{i-1}}{\left(\left(\left(1-\alpha_{i}\right) b_{i}+\alpha_{i} c_{i} x_{i}\right)^{\gamma_{i}}+x_{i-1}^{\gamma_{i}}\right)^{1 / \gamma_{i}}} \\
a_{i}>0, b_{i}>0, c_{i}>0,0 \leq \alpha_{i} \leq 1, \gamma_{i}>1\end{array}$ & $\begin{array}{c}\varepsilon_{x_{i-1}}^{f_{i}}=\frac{\left(\left(1-\alpha_{i}\right) b_{i}+\alpha_{i} c_{i} x_{i}\right)^{\gamma_{i}}}{\left(\left(1-\alpha_{i}\right) b_{i}+\alpha_{i} c_{i} x_{i}\right)^{\gamma_{i}}+x_{i-1}^{\gamma_{i}}} \\
\varepsilon_{x_{i}}^{f_{i}}=\frac{\alpha_{i} c_{i} x_{i}\left(\left(1-\alpha_{i}\right) b_{i}+\alpha_{i} c_{i} x_{i}\right)^{\gamma_{i}-1}}{\left(\left(1-\alpha_{i}\right) b_{i}+\alpha_{i} c_{i} x_{i}\right)^{\gamma_{i}}+x_{i-1}^{\gamma_{i}}}\end{array}$ \\
\hline
\end{tabular}

In MCA, elasticities are usually considered in terms of total rates $v_{i}=x_{i} f_{i}$ rather than per-capita rates $f_{i}$. In the most general case $v_{i}(\boldsymbol{x})=x_{i} f_{i}(\boldsymbol{x})$, the relationship between the two kinds of elasticity coefficients, using the definition provided by Eq. (4) is

$\varepsilon_{x_{j}}^{f_{i}}=\varepsilon_{x_{j}}^{v_{i}}-\delta_{j}^{i}, \quad i, j=1, \ldots, n$,

where $\delta_{i}^{i}=1$ and $\delta_{j}^{i}=0$ when $j \neq i$. If we use $E_{\boldsymbol{x}}^{v}$ and $\mathcal{E}_{\boldsymbol{x}}^{f}$ to represent the matrices of the total and per-capita elasiticities $\varepsilon_{x_{j}}^{v_{i}}$ and $\varepsilon_{x_{j}}^{f_{i}}$, respectively, this equation becomes

$E_{x}^{v}=\mathcal{E}_{x}^{f}+I$.
In Lotka-Volterra and derivative models (e.g. Rosenzweig and MacArthur, 1963) growth is assume to be a linear function of feeding: the slope parameter $r$ accounts for the direct conversion of ingested resource to population biomass (or, alternatively, population numbers), and the intercept parameter $m$ accounts for biomass losses due to deaths (Table 2). In populations that do not decay at an exponential rate $m$ when resources are absent, a hyperbolic growth model or a metaphysiological approach may be more appropriate. These functions, together with their elasticities are listed in Table 2.

Finally, although we do not consider the more general situation here, in addition to its dependence

Table 2

Growth functions

\begin{tabular}{llc}
\hline Name & Form $(i=1,2)$ & Elasticities \\
\hline Linear growth (Lotka-Volterra) & $g_{i}\left(f_{i}\right)=r_{i} f_{i}-m_{i}, \quad r_{i}>0, m_{i}>1$ & $\varepsilon_{f_{i}}^{g_{i}}=\frac{r_{i} f_{i}}{m_{i}-r_{i} f_{i}}$ \\
Hyperbolic growth (Getz, 1991, 1993, 1994) & $g_{i}\left(f_{i}\right)=r_{i}\left(1-\frac{m_{i}}{f_{i}}\right), \quad r_{i}>0,0<m_{i}<\max f_{i}$ & $\varepsilon_{f_{i}}^{g_{i}}=\frac{m_{i}}{f_{i}-m_{i}}$ \\
$\begin{array}{l}\text { Metaphysiological growth (Getz } \\
\text { and Owen-Smith, 1999) }\end{array}$ & $g_{i}\left(f_{i}\right)=r_{i} f_{i}-m_{i}-\frac{q_{i}}{f_{i}}, \quad r_{i}>0, m_{i}>0, q_{i}>0$ & $\varepsilon_{f_{i}}^{g_{i}}=\frac{r_{i} f_{i}^{2}+q_{i}}{r_{i} f_{i}^{2}-m_{i} f_{i}-q_{i}}$ \\
\hline
\end{tabular}


directly on feeding, the growth function may also depend directly on other state variables (Westerhoff and Van Dam, 1987; Westerhoff et al., 2002). Growth may even depend on an external forcing function that, for example, represents a seasonal signal or a climatic trend (e.g. global warming) driving the model.

\section{Control theorems}

Our purpose in this section is to present the main technical results of a control analysis of a general trophic chain model with respect to perturbations in the feeding and growth functions. The proofs are relegated to the appendices. In the next section, we demonstrate how these results can be applied to assessing the relative importance of different feeding and growth functions in determining the steady-state levels (or more appropriately in some cases, our representations for the long term average levels) of the various populations in the trophic chain.

The theorems stated below allow us to express the growth coefficients in terms of elasticity coefficients and other specially defined matrices. In particular, with respect to a steady-state solution $\hat{\boldsymbol{x}}>\mathbf{0}$ (the inequality applies element-wise with respect to the vector $\mathbf{0}=$ $\left.(0, \ldots, 0)^{\prime}\right)$ to Eq. $(1)$, we define matrices $\hat{X}_{\mathrm{D}}, \hat{T}_{\mathrm{D}}$, and $\hat{G}_{\mathrm{D}}$ to be diagonal matrices whose diagonal elements are respectively the vectors $\hat{\boldsymbol{x}}, \hat{\boldsymbol{f}}=\boldsymbol{f}(\hat{\boldsymbol{x}})$ and $\hat{\boldsymbol{g}}=\boldsymbol{g}(\hat{\boldsymbol{f}})$. Also, we do not limit our analysis to the particular case $f_{i}\left(x_{i-1}, x_{i}\right)$, but assume the general dependence $f_{i}(\hat{\boldsymbol{x}})$. On the other hand, we are more specific about the form of the growth functions $g_{i}$ and, in particular, our first theorem relates to systems in which growth is a linear function of extraction, viz. $g_{i}\left(f_{i}\right)=r_{i} f_{i}-m_{i}, i=1, \ldots, n$.

\subsection{Connectivity Theorem I: linear growth functions}

Consider the $n$-species trophic chain

$$
\frac{\mathrm{d} x_{i}}{\mathrm{~d} t}=r_{i} f_{i} x_{i}-f_{i+1} x_{i+1}-m_{i} x_{i}, \quad i=1, \ldots, n,
$$

where $r_{i}$ and $m_{i}$ are positive constants and the undefined quantity $f_{n+1} x_{n+1}$ is fixed at some constant nonnegative value symbolically represented by $h=\hat{f}_{n+1} \hat{x}_{n+1}$. Initially, conditions are such that all species densities are initially positive and the structure of the feeding rate functions ensures that species remain positive. Define

$$
R=\left(\begin{array}{cccccc}
r_{1} & -1 & 0 & \cdots & 0 & 0 \\
0 & r_{2} & -1 & \cdots & 0 & 0 \\
0 & 0 & r_{3} & \ddots & 0 & 0 \\
\vdots & \vdots & \vdots & \ddots & \ddots & \vdots \\
0 & 0 & 0 & \cdots & r_{n-1} & -1 \\
0 & 0 & 0 & \cdots & 0 & r_{n}
\end{array}\right) .
$$

For all $r_{i}>0, i=1, \ldots, n$, this matrix $R$ is invertible. Consequently

$\hat{S}=\left(R \hat{T}_{\mathrm{D}} \hat{X}_{\mathrm{D}}\right)^{-1} M_{\mathrm{D}} \hat{X}_{\mathrm{D}}$

exists, where $M_{\mathrm{D}}$ is a diagonal matrix with diagonal elements $m_{i}$ (the other diagonal matrices $\hat{X}_{\mathrm{D}}$ and $\hat{T}_{\mathrm{D}}$ as defined above) and then the matrix $\mathcal{C}_{f}^{x}$ of feeding rate control coefficients associated with a particular steady-state $\hat{\boldsymbol{x}}>\mathbf{0}$ is given by

$\mathcal{C}_{f}^{x}\left(I+\mathcal{E}_{x}^{f}-\hat{S}\right)=-I$

whenever the matrix $\left(I+\mathcal{E}_{x}^{f}-\hat{S}\right)$ is invertible.

A proof of this theorem is given in Appendix A.

\section{Notes}

1. If, as in MCA, we had regarded perturbations to the total rates $v_{i}=f_{i} x_{i}$ rather than to the per-capita rates $f_{i}$, then, from Eq. (7), our connectivity relationship (10) would have been written as

$\mathcal{C}_{f}^{x}\left(E_{x}^{v}-\hat{S}\right)=-I$.

The difference between the result presented here and the standard MCA connectivity result $\mathcal{C}_{f}^{x} E_{x}^{v}=$ $-I$ is that in our treatment here the matrix $\hat{S}$ arises as a correction matrix because the rates $m_{i} x_{i}$ are themselves not perturbed. If the maintenance related parameters $m_{i}$ are zero then, in effect, the matrix $\hat{S}$ is 0 , although a more detailed derivation of the result than the one we present is required for the case $m_{i}=0$.

2. At any steady state $\hat{\boldsymbol{x}}>\mathbf{0}$ obtained for the case $h=f_{n+1} x_{n+1}=0$, provided the parameters 
$m_{i}>0$, we have $\left(r_{i} \hat{f}_{i} \hat{x}_{i}-\hat{f}_{i+1} \hat{x}_{i+1}\right)=m_{i} \hat{x}_{i}$, $i=1, \ldots, n$. These relationships imply that the matrix $\hat{S}$ has the form

$$
\hat{S}=\left(\begin{array}{cccc}
\frac{m_{1} \hat{x}_{1}}{r_{1} \hat{f}_{1} \hat{x}_{1}} & \frac{m_{2} \hat{x}_{2}}{r_{1} r_{2} \hat{f}_{1} \hat{x}_{1}} & \cdots & \frac{m_{n-1} \hat{x}_{n-1}}{r_{1} r_{2} \cdots r_{n-1} \hat{f}_{1} \hat{x}_{1}} \\
0 & \frac{m_{2} \hat{x}_{2}}{r_{2} \hat{f}_{2} \hat{x}_{2}} & \cdots & \frac{m_{n-1} \hat{x}_{n-1}}{r_{2} r_{3} \cdots r_{n-1} \hat{f}_{2} \hat{x}_{2}} \\
\vdots & \vdots & \ddots & \vdots \\
0 & 0 & \cdots & \frac{m_{n-1} \hat{x}_{n-1}}{r_{n-1} \hat{f}_{n-1} \hat{x}_{n-1}} \\
0 & 0 & \cdots & 0
\end{array}\right.
$$

Further, it is easily seen that all the elements of $\hat{S}$ are positive and that all rows of $\hat{S}$ sum to 1 (also, see point 3 below).

3. This connectivity theorem applies more generally to the equation

$\frac{\mathrm{d} \boldsymbol{x}}{\mathrm{d} t}=R X_{\mathrm{D}} \cdot \boldsymbol{f}-M_{\mathrm{D}} \cdot \boldsymbol{x}$

for invertible, but otherwise arbitrary $R$. Defining $\mathbf{1}=(1, \ldots, 1)^{\prime}$, the steady-state equation associated with this model (note in this model $f_{n+1} x_{n+1}=0$ ), as commented under Note 2, satisfies

$\hat{S} \cdot \mathbf{1}=\mathbf{1}$

from which it follows that

$\mathcal{C}_{f}^{x} \mathcal{E}_{x}^{f} \cdot \mathbf{1}=-1$.

More specifically, the latter in detail is

$\sum_{j=1}^{n} \sum_{k=1}^{n} c_{f_{j}}^{x_{i}} \varepsilon_{x_{k}}^{f_{j}}=-1, \quad i=1, \ldots, n$

which relates to the effects of all $x_{k}$ on a particular $x_{i}$ through all functions $f_{j}$.

\subsection{Connectivity Theorem II: nonlinear growth functions}

The matrices $\mathcal{C}_{f}^{x}$ of extraction rate control coefficients and $\mathcal{C}_{g}^{x}$ of growth rate control coefficients for the trophic chain

$$
\frac{\mathrm{d} x_{i}}{\mathrm{~d} t}=g_{i}\left(f_{i}\right) x_{i}-f_{i+1} x_{i+1}, \quad i=1, \ldots, n
$$

$$
\left.\begin{array}{c}
\frac{m_{n} \hat{x}_{n}}{r_{1} r_{2} \cdots r_{n-1} \hat{f}_{1} \hat{x}_{1}} \\
\frac{m_{n} \hat{x}_{n}}{r_{2} r_{3} \cdots r_{n-1} \hat{f}_{2} \hat{x}_{2}} \\
\vdots \\
\frac{m_{n} \hat{x}_{n}}{r_{n-1} \hat{f}_{n-1} \hat{x}_{n-1}} \\
\frac{m_{n} \hat{x}_{n}}{r_{n} \hat{f}_{n} \hat{x}_{n}}
\end{array}\right) .
$$

for constant $h=f_{n+1} x_{n+1} \neq 0$, are given by solutions to the equation

$\mathcal{C}_{f}^{x}\left(\mathcal{E}_{x}^{f}+\left(\mathcal{E}_{f}^{g}-T\right)^{-1}(I-T)\right)=-I$

and

$\mathcal{C}_{g}^{x}\left(\left(\mathcal{E}_{f}^{g}-T\right) \mathcal{E}_{x}^{f}+I-T\right)=-I$.

At some steady-state $\hat{\boldsymbol{x}}>\mathbf{0}$ (the inequality applies element-wise with respect to the vector $\mathbf{0}=$ $\left.(0, \ldots, 0)^{\prime}\right)$, where

$T=\left(\begin{array}{ccccc}0 & 1 & 0 & \cdots & 0 \\ 0 & 0 & 1 & \cdots & 0 \\ \vdots & \vdots & \vdots & \ddots & \vdots \\ 0 & 0 & 0 & \cdots & 1 \\ 0 & 0 & 0 & \cdots & 0\end{array}\right)$,

provided the matrices $\left(\mathcal{E}_{f}^{g}-T\right)$ and $\left(\left(\mathcal{E}_{f}^{g}-T\right) \mathcal{E}_{x}^{f}\right.$ $+I-T)$ are invertible.

A proof of this theorem is given in Appendix B.

\section{Notes}

1. From Eqs. (13) and (14), it follows that

$$
\mathcal{C}_{g}^{x}=\mathcal{C}_{f}^{x}\left(\mathcal{E}_{f}^{g}-T\right)^{-1} \text {. }
$$

2. If $h=0$, then at a steady state satisfying $x_{n}>0$ it follows that $g_{n}\left(f_{n}\right)=0$, which implies that the elasticity coefficient $\varepsilon_{f_{n}}^{g_{n}}$ is undefined. The theorem, however, holds for arbitrarily small $h$. Further, the 
results are not explicitly dependent on the value of $h$, but only on the values of $\hat{\boldsymbol{x}}$ as influenced by $h$. Thus, the theorem also holds at $h=0$.

3. In matrix notation, recalling $\boldsymbol{x}=\left(x_{1}, \ldots, x_{n}\right)^{\prime}$ and $\boldsymbol{X}_{\mathrm{D}}$ is a diagonal matrix with elements $x_{i}, i=1, \ldots, n$ on the diagonal, for the case $h=f_{n+1} x_{n+1}=0$ our general Eq. (1) can be expressed more generally in terms of the feeding and growth functions $\boldsymbol{f}(\boldsymbol{x})$ and $\boldsymbol{g}(\boldsymbol{f})$ and an $n \times n$ matrix of constants $T$ as

$\frac{\mathrm{d} \boldsymbol{x}}{\mathrm{d} t}=G_{\mathrm{D}} \boldsymbol{x}-T X_{\mathrm{D}} \boldsymbol{f}$,

where the diagonal elements of the diagonal matrix $G_{\mathrm{D}}$ are the functions $g_{i}\left(f_{i}\right)$. The special case of Eq. (12) is obtained by assuming $T$ is the upper diagonal matrix given above. For general $T$, however, the theorem generalizes to

$\mathcal{C}_{f}^{x}\left(\mathcal{E}_{x}^{f}+\left(\mathcal{E}_{f}^{g}-\hat{L}\right)^{-1}(I-\hat{L})\right)=-I$

and

$\mathcal{C}_{g}^{x}\left(\left(\mathcal{E}_{f}^{g}-\hat{L}\right) \mathcal{E}_{x}^{f}+I-\hat{L}\right)=-I$,

where $\hat{L}=\left(\hat{X}_{\mathrm{D}} \hat{G}_{\mathrm{D}}\right)^{-1} T \hat{X}_{\mathrm{D}} \hat{T}_{\mathrm{D}}$. In this more general case, however, the matrix $\hat{L}$ in the above expression cannot be replaced by the matrix $T$, as it can when $T$ is upper diagonal with unit entries, as defined in the theorem.

4. When the growth rate is simply proportional to the feeding rate, then $\mathcal{E}_{f}^{g}=I$, and the theorem reduces to

$\mathcal{C}_{f}^{x}\left(\mathcal{E}_{x}^{f}+I\right)=-I$,

provided the matrix $\left(\mathcal{E}_{x}^{f}+I\right)$ is invertible.

The two connectivity theorems demonstrate that each control coefficient is expressed by the corresponding element in the inverse of a matrix that essentially is the sum of the identity matrix and the matrix of elasticity coefficients, corrected for losses not balanced in the equations (viz. in Connectivity Theorem I, natural mortality flows to detritus pool that is not included in the model, or, in Connectivity Theorem II, losses not explicitly accounted for in modeling the growth rate as a function of the feeding rate). Further, the theorems elucidate how control in a trophic chain depends on the feeding and growth rates of all populations in the chain rather than by just the population above and below the population of interest. Calculating the actual values of the elasticity coefficients (i.e. the elements of $\mathcal{E}_{x}^{f}$ and $\mathcal{E}_{f}^{g}$ ) and of the elements of $\hat{S}$ (linear case) and of $\hat{L}$ (nonlinear case) requires knowing the values of steady-state population densities $\hat{\boldsymbol{x}}$. For specific systems, these may be obtained experimentally, while fair estimates of elasticity values may be obtained by guessing the power dependence of functions on variables in the neighborhood of the steady state. Also, for simple systems (see below), analytical solutions are possible in terms of system parameter values, otherwise numerical solutions are necessary. Numerical solutions decrease the utility of our theorems in the sense that the numerical values for the control coefficient can always be obtained directly from numerical simulations of how the system responds to perturbations in the growth and feeding function parameters. For complex problems, however, the utility of the theorems still remains in allowing us to predict how the distribution of control is affected by changes in the elasticities of the feeding and growth rates.

\section{Two species chains}

Let $x_{1}(t)$ and $x_{2}(t)$ represent the biomass density of a primary producer and consumer in a two species trophic chain, and let $x_{0}$ represent the buffered density of an underlying nutrient or free energy flux (e.g. light) critical for the growth of the primary producer. Our assumption that $x_{0}$ is buffered is just a way of stating that extraction of this underlying resource has no effect on the density $x_{0}$, as perceived by the producer. For systems in which resources are not buffered, we would need to add an equation that describes the pool dynamics of the resource $x_{0}$ leading to the more complex three-species trophic chain.

Focusing on the case of a buffered resource $x_{0}$, our general mathematical description of a two-species trophic chain overlying this resource is

$$
\frac{\mathrm{d} x_{1}}{\mathrm{~d} t}=g_{1}\left(f_{1}\right) x_{1}-f_{2} x_{2}, \quad \frac{\mathrm{d} x_{2}}{\mathrm{~d} t}=g_{2}\left(f_{2}\right) x_{2}-h,
$$

where $g_{1}\left(f_{1}\right)$ and $g_{2}\left(f_{2}\right)$ are per-capita growth func- 
tions, $f_{1}\left(x_{0}, x_{1}\right)$ and $f_{2}\left(x_{1}, x_{2}\right)$ are the per-capita resource extraction functions, and $h \geq 0\left(h \equiv f_{3} x_{3}\right)$ is a rate that allows for extraction of the second species by some process "above" the chain (e.g. harvesting by humans).

\subsection{Linear growth functions}

If we assume that the growth functions have the linear form (Table 2) $g_{i}\left(f_{i}\right)=r_{i} f_{i}-m_{i}$, then the trophic chain Eq. (16) becomes

$$
\begin{aligned}
\frac{\mathrm{d} x_{1}}{\mathrm{~d} t} & =\left(r_{1} f_{1}-m_{1}\right) x_{1}-f_{2} x_{2}, \\
\frac{\mathrm{d} x_{2}}{\mathrm{~d} t} & =\left(r_{2} f_{2}-m_{2}\right) x_{2}-h .
\end{aligned}
$$

In this case, if $h=0$, positive steady-state solutions $\left(\hat{x}_{1}, \hat{x}_{2}\right)^{\prime}$ are simultaneous solutions to the two equations

$$
\begin{aligned}
& r_{2} f_{2}\left(\hat{x}_{1}, \hat{x}_{2}\right)=m_{2} \Rightarrow \hat{f}_{2}=\frac{m_{2}}{r_{2}} \\
& \left(r_{1} f_{1}\left(\hat{x}_{0}, \hat{x}_{1}\right)-m_{1}\right) \hat{x}_{1} \\
& \quad=f_{2}\left(\hat{x}_{1}, \hat{x}_{2}\right) \hat{x}_{2} \Rightarrow \hat{f}_{1}=\frac{m_{1} \hat{x}_{1}+\hat{f}_{2} \hat{x}_{2}}{r_{1} \hat{x}_{1}} .
\end{aligned}
$$

For this system

$$
R=\left(\begin{array}{cc}
r_{1} & -1 \\
0 & r_{2}
\end{array}\right)
$$

and (see Eq. (9) and Note 2 in Section 3.1):

$$
\hat{S}=\left(\begin{array}{cc}
\frac{r_{2} m_{1} \hat{x}_{1}}{r_{2} m_{1} \hat{x}_{1}+m_{2} \hat{x}_{2}} & \frac{m_{2} \hat{x}_{2}}{r_{2} m_{1} \hat{x}_{1}+m_{2} \hat{x}_{2}} \\
0 & 1
\end{array}\right) .
$$

Noting that $f_{1}=f_{1}\left(x_{0}, x_{1}\right) \Rightarrow \varepsilon_{x_{2}}^{f_{1}}=0$, the control coefficients in terms of the growth parameters $r_{1}, r_{2}, m_{1}$ and $m_{2}$ and the remaining three elasticity coefficients $\varepsilon_{x_{1}}^{f_{1}}, \varepsilon_{x_{1}}^{f_{2}}$ and $\varepsilon_{x_{2}}^{f_{2}}$ can be calculated by inverting the matrix (see Section 3.1) to obtain the individual solutions

$$
c_{f_{1}}^{x_{1}}=-\frac{\varepsilon_{x_{2}}^{f_{2}}\left(m_{1} r_{2} \hat{x}_{1}+m_{2} \hat{x}_{2}\right)}{\psi_{\text {linear }}\left(\hat{\boldsymbol{f}}, \mathcal{E}_{\boldsymbol{x}}^{f}\right)}
$$

$$
c_{f_{2}}^{x_{1}}=-\frac{m_{2} \hat{x}_{2}}{\psi_{\text {linear }}\left(\hat{\boldsymbol{f}}, \mathcal{E}_{\boldsymbol{x}}^{\boldsymbol{f}}\right)}
$$

$c_{f_{1}}^{x_{2}}=\frac{\varepsilon_{x_{1}}^{f_{2}}\left(m_{1} r_{2} \hat{x}_{1}+m_{2} \hat{x}_{2}\right)}{\psi_{\text {linear }}\left(\boldsymbol{f}, \mathcal{E}_{\boldsymbol{x}}^{f}\right)}$

$$
c_{f_{2}}^{x_{2}}=-\frac{\varepsilon_{x_{1}}^{f_{1}}\left(m_{1} r_{2} \hat{x}_{1}+m_{2} \hat{x}_{2}\right)+m_{2} \hat{x}_{2}}{\psi_{\text {linear }}\left(\boldsymbol{f}, \mathcal{E}_{\boldsymbol{x}}^{f}\right)},
$$

where

$$
\begin{aligned}
\psi_{\text {linear }}\left(\hat{\boldsymbol{f}}, \mathcal{E}_{\boldsymbol{x}}^{f}\right)= & \varepsilon_{x_{1}}^{f_{1}} \varepsilon_{x_{2}}^{f_{2}}\left(m_{1} r_{2} \hat{x}_{1}+m_{2} \hat{x}_{2}\right) \\
& +\left(\varepsilon_{x_{1}}^{f_{2}}+\varepsilon_{x_{2}}^{f_{2}}\right) m_{2} \hat{x}_{2} .
\end{aligned}
$$

Also, taking ratios, and using the notation $c_{f_{1} / f_{2}}^{x_{1}}=$ $c_{f_{1}}^{x_{1}} / c_{f_{2}}^{x_{1}}$ it follows that

$c_{f_{1} / f_{2}}^{x_{1}}=\varepsilon_{x_{2}}^{f_{2}}\left(1+\frac{m_{1} r_{2} \hat{x}_{1}}{m_{2} \hat{x}_{2}}\right)$

and that

$c_{f_{2} / f_{1}}^{x_{2}}=-\frac{\varepsilon_{x_{1}}^{f_{1}}}{\varepsilon_{x_{1}}^{f_{2}}}\left(1+\frac{m_{2} \hat{x}_{2}}{\varepsilon_{x_{1}}^{f_{1}}\left(m_{1} r_{2} \hat{x}_{1}+m_{2} \hat{x}_{2}\right)}\right)$.

If $m_{1}$ is negligible (i.e. if the overwhelming cause of prey death is predation), then

$c_{f_{1} / f_{2}}^{x_{1}} \approx \varepsilon_{x_{2}}^{f_{2}}$

and

$c_{f_{2} / f_{1}}^{x_{2}} \approx-\frac{\varepsilon_{x_{1}}^{f_{1}}+1}{\varepsilon_{x_{1}}^{f_{2}}}$.

Verbal interpretation of these results are given in Section 6.

$$
-I-\mathcal{E}_{\boldsymbol{x}}^{f}+\hat{S}=\left(\begin{array}{cc}
-1-\varepsilon_{x_{1}}^{f_{1}}+\frac{r_{2} m_{1} \hat{x}_{1}}{r_{2} m_{1} \hat{x}_{1}+m_{2} \hat{x}_{2}} & \frac{m_{2} \hat{x}_{2}}{r_{2} m_{1} \hat{x}_{1}+m_{2} \hat{x}_{2}} \\
-\varepsilon_{x_{1}}^{f_{2}} & -\varepsilon_{x_{2}}^{f_{2}}
\end{array}\right)
$$




\subsection{Hyperbolic growth functions}

If we assume that the growth functions have the hyperbolic form

$g_{i}\left(f_{i}\right)=r_{i}\left(1-\frac{m_{i}}{f_{i}}\right)$,

then the trophic chain equations (16) become

$$
\begin{aligned}
\frac{\mathrm{d} x_{1}}{\mathrm{~d} t} & =r_{1}\left(1-\frac{m_{1}}{f_{1}}\right) x_{1}-f_{2} x_{2}, \\
\frac{\mathrm{d} x_{2}}{\mathrm{~d} t} & =r_{2}\left(1-\frac{m_{2}}{f_{2}}\right) x_{2}-h .
\end{aligned}
$$

In this case, the positive steady-state solutions $\left(\hat{x}_{1}, \hat{x}_{2}\right)^{\prime}$ (more than one may exist, depending on the forms of $f_{1}$ and $f_{2}$ ) are found by solving

$r_{1}\left(1-\frac{m_{1}}{\hat{f}_{1}}\right) \hat{x}_{1}=\hat{f}_{2} \hat{x}_{2}$ and $r_{2}\left(1-\frac{m_{2}}{\hat{f}_{2}}\right) \hat{x}_{2}=h$.

For this system, it follows from Table 2 that the elasticity matrix is given by

$\mathcal{E}_{\boldsymbol{f}}^{\boldsymbol{g}}=\left(\begin{array}{cc}\frac{m_{1}}{\hat{f}_{1}-m_{1}} & 0 \\ 0 & \frac{m_{2}}{\hat{f}_{2}-m_{2}}\end{array}\right)$.

The matrix $T$ is simply

$T=\left(\begin{array}{ll}0 & 1 \\ 0 & 0\end{array}\right)$

so that the inverse $-\mathcal{E}_{x}^{f}-\left(\mathcal{E}_{f}^{g}-T\right)^{-1}(I-T)$ of the control matrix $\mathcal{C}_{f}^{x}$, as stated in Control Coefficient Connectivity Theorem II, can be calculated in terms of the hyperbolic growth parameters $r_{i}$ and $m_{i}$, the steady-state values of the feeding functions $\hat{f}_{i}$, and the feeding function elasticity coefficients $\varepsilon_{x_{j}}^{f_{i}}, i, j=1,2$ to obtain
Inverting this matrix yields the individual control coefficients:

$c_{f_{1}}^{x_{1}}=\frac{m_{1}\left(-\hat{f}_{2}+m_{2}\left(1-\varepsilon_{x_{2}}^{f_{2}}\right)\right)}{\psi_{\text {hyp }}\left(\hat{\boldsymbol{f}}, \mathcal{E}_{\boldsymbol{x}}^{f}\right)}$

$c_{f_{2}}^{x_{1}}=\frac{\hat{f}_{1}\left(\hat{f}_{2}-2 m_{2}\right)-m_{1}\left(\hat{f}_{2}-m_{2}\left(\varepsilon_{x_{2}}^{f_{1}}+2\right)\right)}{\psi_{\mathrm{hyp}}\left(\hat{\boldsymbol{f}}, \mathcal{E}_{\boldsymbol{x}}^{f}\right)}$

$c_{f_{1}}^{x_{2}}=\frac{\varepsilon_{x_{1}}^{f_{2}} m_{1} m_{2}}{\psi_{\text {hyp }}\left(\hat{\boldsymbol{f}}, \mathcal{E}_{\boldsymbol{f}}^{\boldsymbol{x}}\right)}$

$c_{f_{2}}^{x_{2}}=\frac{m_{2}\left(m_{1}\left(1-\varepsilon_{x_{1}}^{f_{1}}\right)-\hat{f}_{1}\right)}{\psi_{\text {hyp }}\left(\hat{\boldsymbol{f}}, \mathcal{E}_{f}^{\boldsymbol{x}}\right)}$,

where $\psi_{\text {hyp }}\left(\hat{\boldsymbol{f}}, \mathcal{E}_{f}^{\boldsymbol{x}}\right)=m_{1} m_{2}\left(1-\varepsilon_{x_{1}}^{f_{1}}-\varepsilon_{x_{1}}^{f_{2}}\left(\varepsilon_{x_{2}}^{f_{1}}+2\right)+\right.$ $\left.\varepsilon_{x_{2}}^{f_{2}}\left(\varepsilon_{x_{1}}^{f_{1}}-1\right)\right)+m_{1} \hat{f}_{2}\left(1-\varepsilon_{x_{1}}^{f_{1}}-\varepsilon_{x_{1}}^{f_{2}}\right)+\hat{f}_{1} \hat{f}_{2}\left(1-\varepsilon_{x_{1}}^{f_{2}}\right)+$ $\hat{f}_{1} m_{2}\left(2 \varepsilon_{x_{1}}^{f_{2}}+\varepsilon_{x_{2}}^{f_{2}}-1\right)$.

Also, taking ratios we have

$c_{f_{2} / f_{1}}^{x_{1}}=\frac{m_{1}\left(\hat{f}_{2}-m_{2}\left(\varepsilon_{x_{2}}^{f_{1}}+2\right)\right)-\hat{f}_{1}\left(\hat{f}_{2}-2 m_{1}\right)}{m_{1}\left(\hat{f}_{2}+m_{2}\left(\varepsilon_{x_{2}}^{f_{2}}-1\right)\right)}$

and that

$c_{f_{2} / f_{1}}^{x_{2}}=\frac{-\hat{f}_{1}+m_{1}\left(1-\varepsilon_{x_{1}}^{f_{1}}\right)}{\varepsilon_{x_{1}}^{f_{2}} m_{1}}$.

In most of the standard models, the feeding rate of the prey does not depend on the density of the predator above it-i.e. $\varepsilon_{x_{2}}^{f_{1}}=0$ (see Table 1). This simplifying assumption does not hold, however, if prey reduce their feeding rate by engaging in predator avoidance behavior (e.g. foraging less regularly and at times when predators are not around). In this case we would expect $f_{1}(\boldsymbol{x})$ to depend negatively on $x_{2}$, in which case the above expressions apply.

In the simpler case, where $\varepsilon_{x_{2}}^{f_{1}}=0$ and we set $h=0$ (see Note 2, Section 3.2) - that is, the predators have no natural enemies-it follows from the above $\left(\mathcal{C}_{\boldsymbol{f}}^{\boldsymbol{x}}\right)^{-1}=\left(\begin{array}{cc}1-\varepsilon_{x_{1}}^{f_{1}}-\frac{\hat{f}_{1}}{m_{1}} & \frac{\hat{f}_{1}\left(2 m_{2}-\hat{f}_{2}\right)+m_{1}\left(\hat{f}_{2}-m_{2}\left(\varepsilon_{x_{2}}^{f_{1}}+2\right)\right)}{m_{1} m_{2}} \\ -\varepsilon_{x_{1}}^{f_{2}} & 1-\varepsilon_{x_{2}}^{f_{2}}-\frac{\hat{f}_{2}}{m_{2}}\end{array}\right)$. 
predator equation that $\hat{f}_{2}=m_{2}$, so that the relative effects of perturbations of the two feeding functions on the steady-state values of the prey and predator reduce to

$c_{f_{2} / f_{1}}^{x_{1}}=\frac{\hat{f}_{1}-m_{1}}{m_{1} \varepsilon_{x_{2}}^{f_{2}}}$

$c_{f_{2} / f_{1}}^{x_{2}}=-\frac{\varepsilon_{x_{1}}^{f_{1}}}{\varepsilon_{x_{1}}^{f_{2}}}-\frac{\hat{f}_{1}-m_{1}}{m_{1} \varepsilon_{x_{1}}^{f_{2}}}$.

Additionally, if we now use the fact (from setting Eq. (20) to 0) that

$\hat{f}_{1}=\frac{m_{1} r_{1} \hat{x}_{1}}{r_{1} \hat{x}_{1}-m_{2} \hat{x}_{2}}$

it follows that (note in the first ratio we have inverted the role of $f_{1}$ and $f_{2}$ )

$c_{f_{1} / f_{2}}^{x_{1}}=\varepsilon_{x_{2}}^{f_{2}}\left(\frac{r_{1} \hat{x}_{1}}{m_{2} \hat{x}_{2}}-1\right)$

and

$c_{f_{2} / f_{1}}^{x_{2}}=-\frac{\varepsilon_{x_{1}}^{f_{1}}}{\varepsilon_{x_{1}}^{f_{2}}}+\frac{1}{\varepsilon_{x_{1}}^{f_{2}}\left(1-\left(r_{1} \hat{x}_{1} / m_{2} \hat{x}_{2}\right)\right)}$.

Verbal interpretation of these results are given in Section 6.

\subsection{Beddington feeding and linear growth}

Further insights can be obtained after specifying the forms of the feeding functions $f_{i}\left(x_{i-1}, x_{i}\right)$ in Eq. (17). In particular, consider feeding functions having the Beddington form (Table 1). In this case the two null-isoclines corresponding to nonzero steady-state solutions to the equations are

$\frac{\mathrm{d} x_{1}}{\mathrm{~d} t}=0 \Rightarrow \hat{x}_{2}=\frac{\left(b_{2}+\hat{x}_{1}\right)\left(a_{1} r_{1} x_{0}-m_{1}\left(b_{1}+c_{1} \hat{x}_{1}+x_{0}\right)\right)}{a_{2}\left(b_{1}+x_{0}+c_{1} \hat{x}_{1}\right)-c_{2}\left(a_{1} r_{1} x_{0}-m_{1}\left(b_{1}+c_{1} \hat{x}_{1}+x_{0}\right)\right)}$

$\frac{\mathrm{d} x_{2}}{\mathrm{~d} t}=0 \Rightarrow \hat{x}_{1}=\frac{\left(m_{2} \hat{x}_{2}+h\right)\left(b_{2}+c_{2} \hat{x}_{2}\right)}{\hat{x}_{2}\left(a_{2} r_{2}-m_{2}\right)-h}$.

Also, the elasticity matrix for the Beddington form (see Table 1) is

$$
\mathcal{E}_{x}^{f}=\left(\begin{array}{cc}
\frac{-c_{1} \hat{x}_{1}}{b_{1}+c_{1} \hat{x}_{1}+x_{0}} & 0 \\
\frac{b_{2}+c_{2} \hat{x}_{2}}{b_{2}+c_{2} \hat{x}_{2}+\hat{x}_{1}} & \frac{-c_{2} x_{2}}{b_{2}+c_{2} \hat{x}_{2}+\hat{x}_{1}}
\end{array}\right) .
$$

Without loss of generality we can scale the units of $x_{i}, i=0,1,2$, and of time $t$ in a way that corresponds to setting the following four parameters to unity: $b_{1}=$ $1, b_{2}=1, r_{1}=1$ and $r_{2}=1$. With this dimensional scaling, setting $h=0$, and reorganization of terms, the null-isocline equations can be simultaneously solved to yield closed form solutions expressed in terms of the seven parameters $a_{i}, c_{i}, m_{i}, i=1,2$ and $x_{0}$. We denote these solutions by

$$
\begin{aligned}
& \hat{x}_{1}=\xi_{1}\left(a_{1}, a_{2}, c_{1}, c_{2}, m_{1}, m_{2}, x_{0}\right) \quad \text { and } \\
& \hat{x}_{2}=\xi_{2}\left(a_{1}, a_{2}, c_{1}, c_{2}, m_{1}, m_{2}, x_{0}\right) .
\end{aligned}
$$

Explicit solutions can be obtained in terms of surds (specifically square roots), which then permits the control parameters to be calculated directly using the identity

$c_{f_{j}}^{x_{i}}=\frac{a_{j}}{\xi_{i}} \frac{\partial \xi_{i}}{\partial a_{j}}, \quad i, j=1,2$.

This was done for the case $m_{1}=0$ and the results obtained compared with the results obtained using Connectivity Theorem I to verify this theorem for the particular case at hand (details of the algebra are not shown, but were calculated using Mathematica 4.0). Specifically, when $b_{1}=1, b_{2}=1, r_{1}=1, r_{2}=1$, and $h=0$, but keeping $m_{1}$ general, Connectivity Theorem I produces the following expressions for the control coefficients (details of the calculations are omitted: expression were obtained using Mathematica 4.0):

$$
\begin{aligned}
c_{f_{1}}^{x_{1}} & =\frac{c_{2}\left(1+x_{0}+c_{1} \hat{x}_{1}\right)\left(m_{1} \hat{x}_{1}+m_{2} \hat{x}_{2}\right)}{m_{2}\left(1+x_{0}+c_{1} \hat{x}_{1}\right)+c_{1} c_{2} \hat{x}_{1}\left(m_{1} \hat{x}_{1}+m_{2} \hat{x}_{2}\right)} \\
c_{f_{2}}^{x_{1}} & =\frac{-m_{2}\left(1+x_{0}+c_{1} \hat{x}_{1}\right)\left(1+\hat{x}_{1}+c_{2} \hat{x}_{2}\right)}{m_{2}\left(1+x_{0}+c_{1} \hat{x}_{1}\right)+c_{1} c_{2} \hat{x}_{1}\left(m_{1} \hat{x}_{1}+m_{2} \hat{x}_{2}\right)}
\end{aligned}
$$

$$
\begin{aligned}
c_{f_{1}}^{x_{2}} & =\frac{\left(1+x_{0}+c_{1} \hat{x}_{1}\right)\left(1+c_{2} \hat{x}_{2}\right)\left(m_{1} \hat{x}_{1}+m_{2} \hat{x}_{2}\right)}{\hat{x}_{2}\left(m_{2}\left(1+x_{0}+c_{1} \hat{x}_{1}\right)+c_{1} c_{2} \hat{x}_{1}\left(m_{1} \hat{x}_{1}+m_{2} \hat{x}_{2}\right)\right)} \\
c_{f_{2}}^{x_{2}} & =\frac{\left(c_{1} m_{1} \hat{x}_{1}^{2}-m_{2} \hat{x}_{2}\left(1+x_{0}\right)\right)\left(1+\hat{x}_{1}+c_{2} \hat{x}_{2}\right)}{\hat{x}_{2}\left(m_{2}\left(1+x_{0}+c_{1} \hat{x}_{1}\right)+c_{1} c_{2} \hat{x}_{1}\left(m_{1} \hat{x}_{1}+m_{2} \hat{x}_{2}\right)\right)} .
\end{aligned}
$$

We now see that the relative importance of the functions $f_{1}$ and $f_{2}$ in controlling populations 1 and 2 is 
given by the expressions

$c_{f_{1} / f_{2}}^{x_{1}}=-\frac{c_{2}\left(m_{1} \hat{x}_{1}+m_{2} \hat{x}_{2}\right)}{m_{2}\left(1+x_{1}+c_{2} \hat{x}_{2}\right)}$

$c_{f_{1} / f_{2}}^{x_{2}}=\frac{\left(1+x_{0}+c_{1} \hat{x}_{1}\right)\left(1+c_{2} \hat{x}_{2}\right)\left(m_{1} \hat{x}_{1}+m_{2} \hat{x}_{2}\right)}{\left(c_{1} m_{1} \hat{x}_{1}^{2}-m_{2} \hat{x}_{2}\left(1+x_{0}\right)\right)\left(1+\hat{x}_{1}+c_{2} \hat{x}_{2}\right)}$.

Note that these expressions imply that whenever the functional response $f_{2}$ is Holling Type II (i.e. pure resource dependence because $c_{2}=0$ ), then $c_{f_{1}}^{x_{1}}=0$ and $c_{f_{1} / f_{2}}^{x_{1}}=0$. At first, it may seem paradoxical that the prey feeding $f_{1}$ has no effect on its own density $x_{1}$, until we recall that the consumer (predator) has a vertical null-isocline when $c_{2}=0$. In this case, it follows that the predator null-isocline Eq. (24) is (recall we have set $b_{1}=1, b_{2}=1, r_{1}=1, r_{2}=1$, and $h=0$ )

$\frac{a_{2} \hat{x}_{1}}{1+\hat{x}_{1}}=m_{2} \Rightarrow \hat{x}_{1}=\frac{m_{2}}{a_{2}-m_{2}}$.

Thus, for the case where predator interference competition is absent $\left(c_{1}=0\right)$, the prey feeding rate $\left(f_{1}\right)$ has no influence on the prey density $\left(x_{1}\right)$ and consequently the corresponding control coefficient is zero $\left(c_{f_{1}}^{x_{1}}=0\right)$.

For general $c_{2}$, the predator null-isocline Eq. (24) for the case $b_{1}=1, b_{2}=1, r_{1}=1, r_{2}=1$, and $h=0$ can be rewritten as

$\frac{c_{2} \hat{x}_{2}}{\hat{x}_{1}}=\frac{\left(a_{2}-m_{2}\right)}{m_{2}}-\frac{1}{\hat{x}_{1}}$.

If we now assume that the overwhelming number of prey deaths are due to predation (i.e. $m_{1} \approx 0$ ) then the prey null-isocline Eq. (23) reduces to

$\hat{x}_{2}=\frac{\left(1+\hat{x}_{1}\right) a_{1} x_{0}}{a_{2}\left(1+c_{1} x_{1}+\hat{x}_{0}\right)-c_{2} a_{1} x_{0}}$.

The coefficient denoting the relative control by predator and prey feeding rates on prey density reduces to

$c_{f_{1} / f_{2}}^{x_{1}}=-\frac{c_{2} \hat{x}_{2}}{1+x_{1}+c_{2} \hat{x}_{2}}=\varepsilon_{x_{2}}^{f_{2}}$

which provides no additional insight compared with Eq. (18). For this case, however,

$$
\begin{aligned}
c_{f_{1} / f_{2}}^{x_{2}} & =-\frac{\left(1+x_{0}+c_{1} \hat{x}_{1}\right)\left(1+c_{2} \hat{x}_{2}\right)}{\left(1+x_{0}\right)\left(1+\hat{x}_{1}+c_{2} \hat{x}_{2}\right)} \\
& =-\left(1+\frac{c_{1} \hat{x}_{1}}{1+x_{0}}\right)\left(1-\frac{\hat{x}_{1}}{1+\hat{x}_{1}+c_{2} \hat{x}_{2}}\right) .
\end{aligned}
$$

Using Eq. (26), it now follows that

$c_{f_{1} / f_{2}}^{x_{2}}=-\left(1+\frac{c_{1} \hat{x}_{1}}{1+x_{0}}\right)\left(1-\frac{m_{2}}{a_{2}}\right)$.

Note that the denominator of the Beddington feeding function in this case is $\left(1+\hat{x}_{0}+c_{1} \hat{x}_{1}\right)$. Thus, the first term in the above expression for $c_{f_{1} / f_{2}}^{x_{2}}$ is increased from unity in proportion to the ratio of inefficiencies in resource exploitation due to intraspecific competition $\left(c_{1} x_{1}\right)$ and due to resource limitations $\left(\left(1+x_{0}\right)\right.$ - the value of 1 appears in this term because in this case $\left.b_{1}=1\right)$. The second term in the above expression for $c_{f_{1} / f_{2}}^{x_{2}}$ is decreased from unity by the ratio of the predator metabolic breakeven value $\left(m_{2}\right)$ to the predator maximum feeding rate $\left(a_{2}\right)$.

\subsection{Beddington feeding and hyperbolic growth}

For systems with specific growth and feeding functions, general results are difficult to interpret when the number of free parameters is greater than three or four. This is why, as in the previous section, we resort to analyzing special cases obtained by setting some of the parameters to zero. As the systems become more complex, either because the feeding and growth functions each contain three or more parameters, or the number of interacting species is greater than two or three, numerical methods can be used to obtain insights into questions of control. Here, for purposes of illustration, we consider how numerical methods can be used to analyze control in prey-predator systems with hyperbolic growth functions and Beddington feeding functions.

A prey-predator system with hyperbolic growth (Table 2) and Beddington feeding (Table 1) is modeled by the specific set of equations (i.e. insert the specific growth and feeding functions in Eq. (16))

$$
\begin{aligned}
\frac{\mathrm{d} x_{1}}{\mathrm{~d} t}= & r_{1}\left(1-\frac{m_{1}\left(b_{1}+c_{1} x_{1}+x_{0}\right)}{a_{1} x_{0}}\right) x_{1} \\
& -\frac{a_{2} x_{1}}{b_{2}+c_{2} x_{2}+x_{1}} x_{2}, \\
\frac{\mathrm{d} x_{2}}{\mathrm{~d} t}= & r_{2}\left(1-\frac{m_{2}\left(b_{2}+c_{2} x_{2}+x_{1}\right)}{a_{2} x_{1}}\right) x_{2}-h .
\end{aligned}
$$


Control in this model was investigated with respect to the parameter set

$$
\mathcal{P}:=\left\{a_{1}, a_{2}, b_{1}, b_{2}, c_{1}, c_{2}, h, m_{1}, m_{2}, r_{1}, r_{2}, x_{0}\right\}
$$

using the following parameter values as our baseline for our numerical simulations and calculations:

$\mathcal{P}_{\text {base }}=\{1,1,5,20,2,10,0,0.1,0.5,8,2,100\}$.

Numerical simulations were carried out using a Runge-Kutta 4 method of integration with step size of $\Delta t=1 / 16$ implemented using the software simulation program Berkeley Madonna 8.0.2. For this set of parameters, the equations have a steady-state solution (rounded to four digits)

$\hat{\boldsymbol{x}}=(444.5,42.45)$.

$$
\begin{aligned}
\frac{\mathrm{d} x_{1}}{\mathrm{~d} t} & =0 \Rightarrow x_{2} \\
& =\frac{r_{1}\left(b_{2}+x_{1}\right)\left(a_{1} x_{0}-m_{1}\left(b_{1}+c_{1} x_{1}+x_{0}\right)\right)}{a_{1} a_{2} x_{0}-r_{1} c_{2}\left(a_{1} x_{0}-m_{1}\left(b_{1}+c_{1} x_{1}+x_{0}\right)\right)}
\end{aligned}
$$

$$
\frac{\mathrm{d} x_{2}}{\mathrm{~d} t}=0 \Rightarrow x_{1}=\frac{m_{2} r_{2} x_{2}\left(b_{2}+c_{2} x_{2}\right)}{r_{2} x_{2}\left(a_{2}-m_{2}\right)-a_{2} h} .
$$

2. Numerically calculate and invert the matrix $-\mathcal{E}_{x}^{f}-$ $\left(\mathcal{E}_{f}^{g}-T\right)^{-1}(I-T)$.

Note, the matrix $T$ is specified in the statement of Connectivity Theorem II and, the elasticity matrix $\mathcal{E}_{x}^{f}$ is given by expression (25), and the elasticity matrix for the growth functions is (see Table 2)

$$
\mathcal{E}_{f}^{g}=\left(\begin{array}{cc}
\frac{m_{1}\left(x_{0}+b_{1}+c_{1} \hat{x}_{1}\right)}{a_{1} x_{0}-m_{1}\left(x_{0}+b_{1}+c_{1} \hat{x}_{1}\right)} & 0 \\
0 & \frac{m_{2}\left(\hat{x}_{1}+b_{2}+c_{2} \hat{x}_{2}\right)}{a_{2} \hat{x}_{1}-m_{2}\left(\hat{x}_{1}+b_{2}+c_{2} \hat{x}_{2}\right)}
\end{array}\right) .
$$

As the parameter $h$ increases from 0 to 10.16 , the prey steady-state density increases very slightly to $x_{1}=445.5$, while the predator steady-state density decreases to $x_{2}=21.45$. At $h=10.17$, these steady states are no longer stable. This type of bifurcation to instability is typical of systems subject to constant harvesting (Getz and Haight, 1989).

The control coefficients were calculated with respect to the baseline parameter set $\mathcal{P}_{\text {base }}$, except for $h$ which was set to $h=0.01$. In particular, the control coefficients $c_{f_{1}}^{x_{1}}$ and $c_{f_{1}}^{x_{2}}$ were obtained from Eq. (6) by respectively calculating the change in the prey $\left(x_{1}\right)$ and predator $\left(x_{2}\right)$ densities for a perturbation in the parameter $a_{1}$ from 1 to 1.00001 . Similarly, the control coefficients $c_{f_{2}}^{x_{1}}$ and $c_{f_{2}}^{x_{2}}$ were obtained for a perturbation in the parameter $a_{2}$ from 1 to 1.00001 to yield the control matrix

$\mathcal{C}_{f}^{x}=\left(\begin{array}{cc}1.11775 & -0.01406 \\ 1.17071 & 2.08027\end{array}\right)$.

The same values were obtained directly from the formula given in Connectivity Theorem II, using Mathematica 4.0 to

1. Calculate the steady-state values from the two null-isocline equations
The values so obtained were identical to five decimal places with the values of the matrix $\mathcal{C}_{f}^{x}$ given above.

As the value of $h$ increases from 0 to 10.16 , at which point the equations are no longer stable, the value of the control coefficients $c_{f_{1}}^{x_{2}}$ and $c_{f_{2}}^{x_{2}}$ slowly increase until they have doubled their value between $h=8$ and $h=9$. Beyond this point the values increase hyperbolically to become infinite when the bifurcation to instability occurs. On the other hand, the control coefficients $c_{f_{1}}^{x_{1}}$ and $c_{f_{2}}^{x_{1}}$ slowly decrease with the first coefficient hardly changing in value of over the whole interval until within 0.0005 of the value of $h$ at which bifurcation to unstable equilibria occurs.

\section{Discussion}

The value of analyses are limited by the degree to which the models of the processes being investigated capture the essence of those processes. Consequently, MCA may be intrinsically more powerful than the TCA presented here merely because biochemical kinetic models are more able to capture the dynamics of biochemical networks than population models are able to capture the dynamics of foodwebs. Foodwebs 
are far more complicated than biochemical networks because stoichiometric principles cannot be applied in quite the same way (Giersch, 1995)—although stoichiometric principles have been invoked in understanding growth rates and $\mathrm{C}: \mathrm{N}: \mathrm{P}$ ratios in the tissues of organisms as a function of the relative availability of phosphorus in various ecosystems (see Elser et al., 2000 and the references therein). An additional complicating factor is that individual animals have a vastly richer repertoire of behaviors than molecules.

At this time, the TCA methods presented here provide the most coherent quantitative theory for analyzing distributed control in trophic chains. Thus, we need to use this tool to obtain whatever insights we can into questions that have interested foodweb ecologist for the past $40+$ years. For example, the numerical study in the previous section illustrates how TCA provides a complete characterization of the impact of perturbations to feeding rates on the long term densities of populations at various levels in a trophic chain (i.e. after the transient effects of the perturbations have disappeared). Thus, in the specific 2-trophic interaction analyzed in Section 4.4, the resulting four numerical values (rounded to two d.p.) - that is $c_{f_{1}}^{x_{1}}=1.12, c_{f_{2}}^{x_{1}}=-0.01, c_{f_{1}}^{x_{2}}=1.17$, and $c_{f_{2}}^{x_{2}}=2.08$-imply the following:

- A (small) unit increase in the per-capita feeding rate of the prey results in a 112 and $117 \%$ unit increase in the long-term prey and predator densities respectively.

- A (small) unit increase in the per-capita feeding rate of the predator reduces the long-term density of the prey by a mere $1 \%$ of a unit, but results in a $208 \%$ unit increase in the long-term density of the predators.

This information could not be guessed ahead of time, but emerges through TCA analysis because of the nonlinearities in the system.

Beyond providing a method for conducting a global analysis of distributed control of population densities by feeding and growth processes occurring at each level in a trophic chain, TCA also provides a control and elasticity coefficient vocabulary for conceptual discussions of foodwebs. As examples of this, we recast some of the mathematical results obtained for the different cases considered in Section 4, in terms of control and elasticity coefficients.
Case 1 (Prey-predator interactions-linear dependence of growth on feeding). Consider a prey-predator systems in which the per-capita growth rate of the prey in exploiting a buffered (i.e. constant density) resource is proportional to its feeding rate minus losses to predation, and the per-capita growth of the predator is proportional to its feeding rate minus its natural death rate. In such systems, control of the prey feeding rate relative to the predator feeding rate on:

- prey density is equal to the value of the elasticity coefficient of the predator feeding rate with respect to predator density (i.e. equals $\varepsilon_{x_{2}}^{f_{2}}$-interpretation of Eq. (18)) so that, essentially, changes in predator feeding efficiency or levels of satiation have no effect on prey density;

- predator density is negative-with prey feeding exercising positive control and predator feeding negative control-and approximately equal in magnitude when, at steady-state, the prey is satiated but the predator is food limited (i.e. in this case $\varepsilon_{x_{1}}^{f_{2}} \approx 1$ ) (interpretation of Eq. (19)).

Case 2 (Prey-predator interactions-hyperbolic dependence of growth on feeding). Consider preypredator systems in which the per-capita growth rates of both the prey and predators populations are hyperbolic functions (see Table 2) of their respective per-capita feeding rates. In such systems, the relative control of prey and predator feeding rates is negative on:

- prey density and is proportional to the elasticity coefficient of the predator feeding rate with respect to predator density multiplied by a factor representing the excess of the ratio of the gross resource conversion rate of the prey (i.e. $r_{1} \hat{x}_{1}$ ) to the metabolic maintenance rate of the predator (i.e. $m_{2} \hat{x}_{2}$ ) (interpretation of Eq. (21)).

- predator density and is proportional to the ratio of the prey and the predator elasticities with respect to prey density with a correction to this amount equal to the inverse of the product of the elasticity of the prey and one minus the ratio of the gross resource conversion rate of the prey (i.e. $\left.r_{1} \hat{x}_{1}\right)$ to the metabolic maintenance rate of the predator (i.e. $m_{2} \hat{x}_{2}$ ) (interpretation of Eq. (22)). 
We cannot apply the theory developed here directly to a trophic cascade hypothesis that posits the idea that each trophic level of a foodweb is inversely related in magnitude to the trophic level above and directly in magnitude to the trophic level below. The reason is that the trophic levels are state variables rather than processes or parameters that affect processes, so a change in their density represents a perturbation from steady state that ultimately leads to a return to the original steady state. Thus any trophic cascade hypothesis should be couched in terms of perturbations to process rates as a whole or parameter values that determine process rates. (An implementation of this would be if one population is replaced by another due to anthropogenic or natural causes, such as mutations, where the feeding or growth rates of the new population are different from those of the old population.)

From the theory presented in this paper, it is clear that a way to couch a trophic hypothesis consistent with the theory is to articulate it in terms of how perturbations to processes at one level in the trophic chain alter trophic levels not directly linked by that process. Thus, for example, one might speculate how an increase in per-capita carnivore consumption rates will affect the density of plants eaten by the intervening herbivore population. A full analysis of this question is beyond the scope of this introductory presentation.

\section{Conclusion}

Metabolic control analysis provides a powerful tool for understanding and manipulating metabolic pathways embodied through biochemical networks (Fell, 1996; Snoep et al., 2002; Ortega et al., 2002). By any measure, organisms are considerably more complex than molecules. Thus, we cannot expect a relatively compact system of ordinary differential equationssuch as Eq. (2) or (1) - to provide anywhere as precise a description of an ecological network, as it can of a biochemical network. Concomitantly, we should not expect control analysis applied to models of foodwebs to be nearly as powerful a tool as it is in biochemical network analysis. Nevertheless, control analysis provides a useful tool, perhaps more useful than any other tool currently available, for understanding how different processes in foodwebs and ecosystems influence populations not directly impacted by those processes.
In other words, despite the limitations in applying any analytical methods to systems as complex as foodwebs, control analysis has much to offer ecologists.

First, control analysis provides a framework for assessing the degree to which any selected process, conceptualized at the level of resolution of the system description (in our case feeding and growth functions), controls steady-state values of the system. It provides understanding of how local processes translate through their elasticity coefficients into global effects. In the case of systems that vary over time, provided the coefficient of variation is relatively small, the results obtained here may be interpreted in terms of long term population averages over time and space rather than steady states that do not exist.

Second, even if the background environment fluctuates on a seasonal basis (i.e. process parameters are periodic functions of time), then control analysis provides a first approximation to assessing the degree to which each factor controls the long term average values for each population. The smaller the fluctuations, the more valid this approximation. Obviously, if fluctuations are relatively large compared with the average values (i.e. the coefficient of variation is close to or greater than 1), then nonlinear effects degrade approximations of average values as represented by a deterministic model (i.e. a certainty equivalence principle fails because of the nonlinear nature of the model-e.g. Getz, 1976).

Third, and most importantly, the value of control analysis applied to foodwebs or ecosystems critically depends on the validity of the equations used to model the systems of interest. Thus, as better models and equations are formulated in the future, the methods discussed here can be applied to these new equations to obtain an assessment of the relative importance of processes modeled by those equations that determine the long term average values of system variables. Note that analytical results for states of systems varying with time, even though more difficult to express than for systems at steady state, have been obtained in a limited way for oscillating biochemical networks and even networks relaxing to their steady-state values over time (Heinrich and Reder, 1991; Kholodenko et al., 1997; Reijenga et al., 2002).

Trophic chains provide a particularly convenient structure for connectivity theorems because the number of feeding and growth functions is equal to the 
number of species in the chain. This results in square control matrices that usually have inverses. More complex webs in which individuals feed on more than one species or are the prey of more than one predator, or webs that lack a well-defined trophic structure because of omnivory or mutual predation (e.g. large individuals in a two species interaction may eat the small individuals of the other species, as is the case for interactions of some piscivorous fish) often require multiple subscripts to conveniently characterize the foodweb (e.g. see Giersch, 1995). This increase in notational complexity makes the development and statement of theorems more complicated. Such impediments can be overcome in developing theorems for more general networks, as we have done elsewhere (Westerhoff et al., 2002, in preparation), but at the expense of some transparency associated with the simpler characterization of a trophic chain compared with a general foodweb.

Finally, in a nutshell, we have derived tools for analyzing control and trophic chains, as well as demonstrated how these tools provide a vocabulary for talking about control in trophic chains. This vocabulary allows us to refine the concept of top-down and bottom-up control in trophic chains and to discuss less ambiguously than before how the relative strengths of control by the various processes are distributed throughout the chain or network.

\section{Acknowledgements}

We are greatly indebted to the Stellenbosch Institute for Advanced Study (STIAS) and their sponsors for support of this work. We thank Mary Power, Sebastian Schreiber, Peter Baxter, Chris Wilmers, and two anonymous reviewers for comments.

\section{Appendix A. Proof of Connectivity Theorem I}

We prove the theorem for the more general system of equations (see Note 3 after statement of the theorem)

$$
\frac{\mathrm{d} x_{i}}{\mathrm{~d} t}=\sum_{k=1}^{n} r_{i k} f_{k} x_{k}-m_{i} x_{i}, \quad i=1, \ldots, n,
$$

where the matrix $R$ of elements $r_{i j}, i, j=1, \ldots, n$, yields Eq. (8) when it has the special form given in the statement of the theorem. In the above equation replace the functions $f_{i}$ by $\lambda_{i} f_{i}$ and consider perturbations of $\lambda_{i}$ in the neighborhood of 1 and of the steady-state solution $\hat{\boldsymbol{x}}$ : i.e. we are interested in the approximations

$\hat{\boldsymbol{x}}\left(\lambda_{j}\right) \approx \hat{\boldsymbol{x}}(1)+\left.\frac{\mathrm{d} \boldsymbol{x}}{\mathrm{d} \lambda_{j}}\right|_{\lambda_{j}=1}\left(\lambda_{j}-1\right)$.

Thus, consider the derivative of the above dynamic equations by $\lambda_{j}$, i.e.

$$
\begin{aligned}
\frac{\mathrm{d}}{\mathrm{d} \lambda_{j}}\left[\frac{\mathrm{d} x_{i}}{\mathrm{~d} t}\right]_{\lambda_{j}=1}= & r_{i j} f_{j} x_{j}+\sum_{k=1}^{n} r_{i k} f_{k} \frac{\mathrm{d} x_{k}}{\mathrm{~d} \lambda_{j}} \\
& +\sum_{k=1}^{n} r_{i k} x_{k} \sum_{l=1}^{n} \frac{\partial f_{k}}{\partial x_{l}} \frac{\mathrm{d} x_{l}}{\mathrm{~d} \lambda_{j}}-m_{i} \frac{\mathrm{d} x_{i}}{\mathrm{~d} \lambda_{j}} .
\end{aligned}
$$

At a steady-state solution $\hat{\boldsymbol{x}}$, the above equation can be written in matrix terms as (recall that $T_{\mathrm{D}}$ is defined to be the diagonal matrix with elements $\hat{f}$ on the diagonal)

$$
\begin{aligned}
{\left[\frac{\mathrm{d}}{\mathrm{d} \lambda} \frac{\mathrm{d} \boldsymbol{x}}{\mathrm{d} t}\right]_{\lambda_{j}=1, x=\hat{\boldsymbol{x}}}=0=} & R \hat{X}_{\mathrm{D}} \hat{T}_{\mathrm{D}}+R \hat{X}_{\mathrm{D}} \hat{T}_{\mathrm{D}} \mathcal{C}_{f}^{\boldsymbol{x}} \\
& +R \hat{X}_{\mathrm{D}} \hat{T}_{\mathrm{D}} \mathcal{E}_{\boldsymbol{x}}^{f} \mathcal{C}_{\boldsymbol{f}}^{\boldsymbol{x}}-M_{\mathrm{D}} \hat{X}_{\mathrm{D}} \mathcal{C}_{f}^{x}
\end{aligned}
$$

Collecting terms in the control coefficient matrix, this reduces to

$\left(R \hat{X}_{\mathrm{D}} \hat{T}_{\mathrm{D}}\left(I+\mathcal{E}_{\boldsymbol{x}}^{f}\right)-M_{\mathrm{D}} \hat{X}_{\mathrm{D}}\right) \mathcal{C}_{f}^{x}=-R \hat{X}_{\mathrm{D}} \hat{T}_{\mathrm{D}}$

If $R$ is invertible, implying that the matrix $\hat{S}=$ $\left(R \hat{X}_{\mathrm{D}} \hat{T}_{\mathrm{D}}\right)^{-1} M_{\mathrm{D}} \hat{X}_{\mathrm{D}}$ exists, this equation becomes

$\left(I+\mathcal{E}_{x}^{f}-\hat{S}\right) \mathcal{C}_{f}^{x}=-I$.

Hence, if the matrix $\left(I+\mathcal{E}_{x}^{f}-\hat{S}\right)$ is invertible, then

$\mathcal{C}_{f}^{x}=-\left(I+\mathcal{E}_{x}^{f}-\hat{S}\right)^{-1}$

and post-multiplying both sides, we obtain the summation Eq. (10), thereby proving the theorem

\section{Appendix B}

Following the same procedure as in Appendix A, we prove the theorem for a general matrix $T$ and 
vector of constants $\boldsymbol{h}=\left(h_{1}, \ldots, h_{n}\right)$, by considering the derivatives of (cf. Eq. (15))

$\frac{\mathrm{d} x_{i}}{\mathrm{~d} t}=x_{i} g_{i}\left(\lambda_{i} f_{i}(\boldsymbol{x})\right)-\sum_{k=1}^{n} t_{i k} x_{k} \lambda_{k} f_{k}(\boldsymbol{x})-h_{i}$

by $\lambda_{j}$ evaluated at $\lambda_{j}=1$ and at the steady-state solution $\hat{\boldsymbol{x}}$ : i.e.

$$
\begin{aligned}
\frac{\mathrm{d}}{\mathrm{d} \lambda_{j}}\left[\frac{\mathrm{d} x_{i}}{\mathrm{~d} t}\right]_{\lambda_{j}=1, \boldsymbol{x}=\hat{\boldsymbol{x}}}=0 \\
=\left[\frac{\mathrm{d}}{\mathrm{d} \lambda_{j}} g_{i}\left(\Lambda_{\mathrm{D}} \boldsymbol{f}(\boldsymbol{x})\right) x_{i}\right. \\
\left.\quad-\frac{\mathrm{d}}{\mathrm{d} \lambda_{j}} \sum_{k=1}^{n} t_{i k} x_{k} \lambda_{k} f_{k}(\boldsymbol{x})\right]_{\lambda_{j}=1, \boldsymbol{x}=\hat{\boldsymbol{x}}}
\end{aligned}
$$

where $\Lambda_{\mathrm{D}}$ is the diagonal matrix with elements $\lambda_{i}$.

First consider

$$
\begin{aligned}
& \left.\frac{\mathrm{d}}{\mathrm{d} \lambda_{j}} \sum_{k=1}^{n} t_{i k} x_{k} \lambda_{k} f_{k}(\boldsymbol{x})\right|_{\lambda=1}=t_{i j} x_{j} f_{j}(\boldsymbol{x}) \\
& \quad+\sum_{k=1}^{n} t_{i k} \frac{\mathrm{d} x_{k}}{\mathrm{~d} \lambda_{j}} f_{k}(\boldsymbol{x})+\sum_{k=1}^{n} t_{i k} x_{k} \sum_{r=1}^{n} \frac{\partial f_{k}}{\partial x_{r}} \frac{\mathrm{d} x_{r}}{\mathrm{~d} \lambda_{j}},
\end{aligned}
$$

which evaluated at $\boldsymbol{x}=\hat{\boldsymbol{x}}$ yields

$$
\begin{gathered}
\left.\frac{\mathrm{d}}{\mathrm{d} \lambda_{j}} \sum_{k=1}^{n} t_{i k} x_{k} \lambda_{k} f_{k}(\boldsymbol{x})\right|_{\lambda=1}=\left(T \hat{X}_{\mathrm{D}} \hat{T}_{\mathrm{D}}\right)_{i j} \\
+\left(T \hat{X}_{\mathrm{D}} \hat{T}_{\mathrm{D}} \mathcal{C}_{\boldsymbol{f}}^{\boldsymbol{x}}\right)_{i j}+\left(T \hat{X}_{\mathrm{D}} \hat{T}_{\mathrm{D}} \mathcal{E}_{\boldsymbol{x}}^{\boldsymbol{f}} \mathcal{C}_{\boldsymbol{f}}^{\boldsymbol{x}}\right)_{i j} .
\end{gathered}
$$

where $T_{\mathrm{D}}$ is the diagonal matrix with diagonal elements $\hat{f}$.

Next consider

$$
\begin{aligned}
& \left.\frac{\mathrm{d}}{\mathrm{d} \lambda_{j}} g_{i}\left(\Lambda_{\mathrm{D}} \boldsymbol{f}(\boldsymbol{x})\right) x_{i}\right|_{\lambda=1}=x_{i} \frac{\partial g_{i}}{\partial f_{j}} f_{j} \\
& +x_{i} \sum_{k=1}^{n} \frac{\partial g_{i}}{\partial f_{k}} \sum_{r=1}^{n} \frac{\partial f_{k}}{\partial x_{r}} \frac{\mathrm{d} x_{r}}{\mathrm{~d} \lambda_{j}}+g_{i} \frac{\mathrm{d} x_{i}}{\mathrm{~d} \lambda_{j}},
\end{aligned}
$$

which evaluated at $\boldsymbol{x}=\hat{\boldsymbol{x}}$ yields

$$
\begin{aligned}
& \left.\frac{\mathrm{d}}{\mathrm{d} \lambda_{j}} g_{i}\left(\Lambda_{\mathrm{D}} \boldsymbol{f}(\boldsymbol{x})\right) x_{i}\right|_{\lambda=1}=\left(\hat{G}_{\mathrm{D}} \hat{X}_{\mathrm{D}} \mathcal{E}_{\boldsymbol{f}}^{\boldsymbol{g}}\right)_{i j} \\
& \quad+\left(\hat{G}_{\mathrm{D}} \hat{X}_{\mathrm{D}} \mathcal{E}_{\boldsymbol{f}}^{\boldsymbol{g}} \mathcal{E}_{\boldsymbol{x}}^{\boldsymbol{f}} \mathcal{C}_{\boldsymbol{f}}^{\boldsymbol{x}}\right)_{i j}+\left(\hat{G}_{\mathrm{D}} \hat{X}_{\mathrm{D}} \mathcal{C}_{\boldsymbol{f}}^{\boldsymbol{x}}\right)_{i j} .
\end{aligned}
$$

The second equation of these Appendices A and B implies that we should equate the right-hand sides of the last two equations to obtain:

$$
\begin{aligned}
& \hat{G}_{\mathrm{D}} \hat{X}_{\mathrm{D}}\left(\mathcal{E}_{f}^{g}+\mathcal{E}_{f}^{g} \mathcal{E}_{x}^{f} \mathcal{C}_{f}^{x}+\mathcal{C}_{f}^{x}\right) \\
& =T \hat{X}_{\mathrm{D}} \hat{T}_{\mathrm{D}}\left(I+\mathcal{C}_{f}^{x}+\mathcal{E}_{x}^{f} \mathcal{C}_{f}^{x}\right) .
\end{aligned}
$$

If $\hat{G}_{\mathrm{D}} \hat{X}_{\mathrm{D}}$ is invertible, and we define $\hat{L}=$ $\left(\hat{G}_{\mathrm{D}} \hat{X}_{\mathrm{D}}\right)^{-1} T \hat{X}_{\mathrm{D}} \hat{T}_{\mathrm{D}}$ then

$\mathcal{E}_{f}^{g}+\mathcal{E}_{f}^{g} \mathcal{E}_{x}^{f} \mathcal{C}_{f}^{x}+\mathcal{C}_{f}^{x}=\hat{L}+\hat{L} \mathcal{C}_{f}^{x}+\hat{L} \mathcal{E}_{x}^{f} \mathcal{C}_{f}^{x}$.

Collecting and rearranging terms contain $\mathcal{C}_{f}^{x}$ implies that

$\left(\left(\mathcal{E}_{f}^{g}-\hat{L}\right) \mathcal{E}_{x}^{f}+(I-\hat{L})\right) \mathcal{C}_{f}^{x}=-\left(\mathcal{E}_{f}^{g}-\hat{L}\right)$.

If the matrices $\left(\mathcal{E}_{f}^{g}-\hat{L}\right)$ and $\left(\left(\mathcal{E}_{f}^{g}-\hat{L}\right) \mathcal{E}_{x}^{f}+(I-\hat{L})\right)$ are invertible, then Eq. (13) is obtained.

Note that for the case $h_{i}=0, i=1, \ldots, n-1$, it follows from the steady-state conditions that

$$
\begin{aligned}
& \frac{1}{x_{i} g_{i}\left(f_{i}(\boldsymbol{x})\right)} \sum_{k=1}^{n} t_{i k} x_{k} f_{k}(\boldsymbol{x})=1, \\
& \quad \text { for all } i=1, \ldots, n-1 .
\end{aligned}
$$

From this, it follows for the trophic cascade which satisfies $t_{i i+1}=1$, and $t_{i j}=0$, when $j \neq i+1$, that if $h_{i}=0, i=1, \ldots, n-1$, that $\hat{L}_{i i+1}=$ $\left(t_{i+1} \hat{x}_{i+1} \hat{f}_{i+1}\right) / \hat{g}_{i} \hat{x}_{i}=1$ and that $\hat{L}_{i j}$ is otherwise 0 . This implies for the special case of the trophic cascade, irrespective of whether or not $h=h_{n}=0$, that $\hat{L}=T$, proving the theorem.

\section{References}

Abrams, P.A., 1994. The fallacies of "ratio-dependent" predation. Ecology 75, 1842-1850.

Arditi, R., Ginzburg, L.R., 1989. Coupling in predator-prey dynamics: ratio-dependence. J. Theor. Biol. 139, 311-326.

Beddington, J.R., 1975. Mutual interference between parasites or predators and its effect on searching efficiency. J. Anim. Ecol. 44, 331-340.

Brett, M.T., Goldman, C.R., 1996. A meta-analysis of the freshwater trophic cascade. Proc. Natl. Acad. Sci. U.S.A. 93, 7723-7726.

Caswell, H., 2000. Matrix Population Models. Sinauer, Sunderland. DeAngelis, B.L., Goldstein, R.A., O’Neill, R.V., 1975. A model for trophic interaction. Ecology 56, 881-982. 
Elser, J.J., Sterner, R.W., Gorokhova, E., Fagan, W.F., Markow, T.A., Cotner, J.B., Harrison, J.F., Hobbie, S.E., Odell, G.M., Weider, L.J., 2000. Biological stoichiometry from genes to ecosystems. Ecol. Lett. 3, 540-550.

Fell, D., 1996. Understanding the Control of Metabolism. Portland Press, London, UK.

Fretwell, S.D., 1977. The regulation of plant communities by the food chains exploiting them. Perspect. Biol. Med. 20, 291-301.

Fretwell, S.D., 1987. Food chain dynamics: the central theory of ecology? Oikos 50, 291-301.

Getz, W.M., 1976. Stochastic equivalents of the linear and Lotka-Volterra system of equations-a general birth-and-death process. Math. Biosci. 29, 235-257.

Getz, W.M., 1984. Population dynamics: a per capita resource approach. J. Theor. Biol. 108, 623-644.

Getz, W.M., 1991. A unified approach to multispecies modeling. Nat. Resour. Model. 5, 393-421.

Getz, W.M., 1993. Metaphysiological and evolutionary dynamics of populations exploiting constant and interactive resources: R-K selection revisited. Evol. Ecol. 7, 287-305.

Getz, W. M., 1994. A metaphysiological approach to modeling ecological populations and communities. In: Levin, S.A. (Ed.), Frontiers in Mathematical Biology. Springer-Verlag, New York, pp. 411-442.

Getz, W. M., 1999. Population and evolutionary dynamics of consumer-resource systems. In: McGlade, J. (Ed.), Theoretical Ecology: Advances in Principles and Applications. Blackwell, Oxford, UK, pp. 194-231.

Getz, W.M., Haight, R.G., 1989. Population Harvesting: Demographic Models of Fish, Forests and Animal Resources. Princeton Monographs in Population Biology. Princeton University Press, Princeton, NJ, $391 \mathrm{pp}$.

Getz, W.M., Owen-Smith, N., 1999. A metaphysiological population model of storage in variable environments. Nat. Resour. Model. 12, 197-230.

Giersch, C., 1988a. Control analysis of metabiolic networks: 1. Homogeneous functions and the summation theorems for control coefficients. Eur. J. Biochem. 174, 509-513.

Giersch, C., 1988b. Control analysis of metabiolic networks: 2. Total differentials and general formulation of the connectivity relations. Eur. J. Biochem. 174, 515-519.

Giersch, C., 1991. Sensitivity analysis of ecosystems: an analytical treatment. Ecol. Model. 35, 131-146.

Giersch, C., 1995. Flux control in ecosystems. Trends Ecol. Evol. $10,245$.

Hairston, N.G., Smith, F.E., Slobodkin, L.B., 1960. Community structure, population control, and competition. Am. Natur. 94, 421-425.

Heinrich, R., Rapoport, T.A., 1974. A linear steady-state treatment of enzymatic chains: general properties, control and effector strength. Eur. J. Biochem. 42, 89-95.

Heinrich, R., Reder, C., 1991. Metabolic control analysis of relaxation processes. J. Theor. Biol. 151, 343-350.

Heinrich, R., Rapoport, S.M., Rapoport, T.A., 1977. Metabolic regulation and mathematical models. Progr. Biophys. Mol. Biol. $32,1-82$.

Hofmeyr, J.-H.S., 2001. Metabolic control analysis in a nutshell. In: Yi, T.-M., Hucka, M., Morohashi, M., Kitano, H. (Eds.),
Proceedings of the 2nd International Conference on Systems Biology, Omnipress, Madison, pp. 291-300.

Hofmeyr, J.-H.S., Cornish-Bowden, A., 1996. Co-response analysis: a new strategy for experimental metabolic control analysis. J. Theor. Biol. 182, 371-3805.

Hofmeyr, J.-H.S., Westerhoff, H.V., 2001. Building the cellular puzzle: control in multi-level reaction networks. J. Theor. Biol. 208, 261-285

Holling, C.S., 1959. Some characteristics of simple types of predation and parasitism. Can. Ent. 91, 385-398.

Kacer, H., Burns, J.A., 1973. The control of flux. Symp. Soc. Exp. Biol. 32, 65-104.

Kahn, D., Westerhoff, H.V., 1991. Control theory of regulatory cascades. J. Theor. Biol. 153, 255-285.

Kholodenko, B.N., Demin, O.V., Westerhoff, H.V., 1997. Control analysis of periodic phenomena in biological systems. J. Phys. Chem. B 101, 2070-2081.

Leslie, P.H., 1948. Some further notes on the use of matrices in population mathematics. Biometrika 35, 213-245.

Lotka, A.J., 1925. The Elements of Physical Biology. Republished in 1956 as: The Elements of Mathematical Biology. Dover, New York, 465 pp.

Matson, P.A., Berryman, A.A., 1992. Ratio-dependent predatorprey theory (eds. of Special Section). Ecology 73, 1529-1566.

Oksanen, L., Fretwell, S.D., Arruda, J., Niemelä, P., 1981. Exploitation ecosystems in gradients of primary productivity. Am. Natur. 118, 240-261.

Oksanen, L., Moen, J., Lundberg, P.A., 1992. The time-scale problem in exploiter-victim models: does the solution lie in ratio-dependent exploitation? Am. Natur. 140, 938-960.

Ortega, F., Acerenza, L., Westerhoff, H.V., Mas, F., Cascante, M., 2002. Product dependence and bifunctionality compromise the ultrasensitivity of signal transduction cascades. Proc. Natl. Acad. Sci. U.S.A. 99, 1170-1175.

Paine, R.T., 1980. Food webs, linkage, interaction strength and community infrastructure. J. Anim. Ecol. 49, 667-685.

Persson, L., Bengtsson, J., Menge, B.A., Power., M.E., 1996. Productivity and consumer regulation-concepts, patterns, mechanisms. In: Polis, G.A., Winemiller, K.O. (Eds.), Food Webs: Integration of Patterns and Dynamics. Chapman and Hall, New York

Polis, A., 1999. Why are parts of the world green? Multiple factors control productivity and the distribution of biomass. Oikos 86 , $3-15$.

Polis, G.A., Strong, D.R., 1996. Food web complexity and community dynamics. Am. Natur. 147, 813-846.

Power, M.E., 1990. Effects of fish on river food webs. Science 250, 811-814.

Power, M.E., 1992. Top-down and bottom-up forces in food webs: do plants have primacy? Ecology 73, 733-746.

Ramos-Jiliberto, R., González-Olivares, E., Bozinovic, F., 2002. Population-level consequences of antipredator behavior: a metaphysiological model based on the functional ecology of the leaf-eared mouse. Theor. Popul. Biol. 62, 63-80.

Reder, C., 1988. Metabolic control theory: a structural approach. J. Theor. Biol. 135, 175-201.

Reijenga, K.A., Westerhoff, H.V., Kholodenko, B.N., Snoep, J.L., 2002. Control analysis for autonomously oscillating biochemical networks. Biophys. J. 82, 99-106. 
Rosenzweig, M.L., MacArthur, R.H., 1963. Graphical representation and stability conditions of predator-prey interactions. Am. Natur. 97, 209-223.

Savageau, M.A., 1976. Biochemical Systems Analysis: A Study of Function and Design in Molecular Biology. Addison-Wesley, London.

Savageau, M.A., 1996. A kinetic formalism for integrative molecular biology: manifestation in biochemical systems theory and use in elucidating design principles for gene circuits. In: Collado-Vides, J., Magasanik, B., Smith, T.F. (Eds.), Integrative Approaches to Molecular Biology. MIT Press, Cambridge, MA, pp. 115-146.

Schuster, S., Westerhoff, H.V., 1999. Modular control analysis of slipping enzymes. BioSystems 49, 1-15.

Schmitz, O.J., Hambäck, P.A., Beckerman, A.P., 2000. Trophic cascades in terrestrial systems: a review of the effects of carnivore removals on plants. Am. Natur. 155, 141-153.

Snoep, J.L., van der Weijden, C.C., Andersen, H.W., Westerhoff, H.V., Jensen, P.R., 2002. DNA supercoiling in Escherichia coli is under tight and subtle homeostatic control, involving gene-expression and metabolic regulation of both topoisomerase I and DNA gyrase. Eur. J. Biochem. 269, 1662-1669.

Swartzman, G.L., Kaluzny, S.P., 1987. Ecological Simulation Primer. Macmillan, New York.

Volterra, V., 1926. Fluctuations in the abundance of species considered mathematically. Nature 118, 558-560.

Wennekers, T., Giersch, C., 1991. Sensitivity analysis of a simple model food chain. Ecol. Model. 54, 265-276.

Westerhoff, H.V., Van Dam, K., 1987. Thermodynamics and Control of Free Energy Transduction. Elsevier, Amsterdam.

Westerhoff, H.V., Getz, W.M., van Verseveld, H.W., Hofmeyr, J.H., Snoep, J.L., 2002. Bioinformatics, cellular flows, and calculation. Ernst Schering Res. Found. Workshop 38, 221243.

Westerhoff, H.V., Hofmeyr, J.H., Snoep, J.L., Getz, W.M. (in preperation). Merging the layers of life: control analysis of ecosystems. 